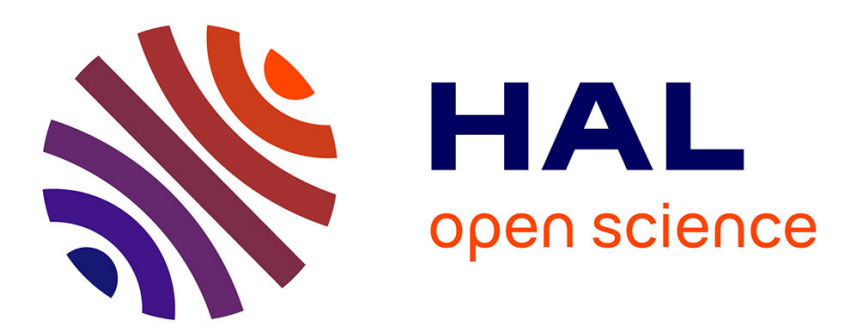

\title{
Geometrical model of spiking and bursting neuron on a mug-shaped branched manifold
}

Mohamed Gheouali, Tounsia Benzekri, René Lozi, Guanrong Chen

\section{To cite this version:}

Mohamed Gheouali, Tounsia Benzekri, René Lozi, Guanrong Chen. Geometrical model of spiking and bursting neuron on a mug-shaped branched manifold. International Journal of Bifurcation and Chaos (IJBC), 2020, 30 (5), pp.2030044. 10.1142/S021812742030044X . hal-02878585

\section{HAL Id: hal-02878585 \\ https://hal.science/hal-02878585}

Submitted on 23 Jun 2020

HAL is a multi-disciplinary open access archive for the deposit and dissemination of scientific research documents, whether they are published or not. The documents may come from teaching and research institutions in France or abroad, or from public or private research centers.
L'archive ouverte pluridisciplinaire HAL, est destinée au dépôt et à la diffusion de documents scientifiques de niveau recherche, publiés ou non, émanant des établissements d'enseignement et de recherche français ou étrangers, des laboratoires publics ou privés. 


\title{
International Journal of bifurcation and Chaos December, 2020
}

\section{Geometrical model of spiking and bursting neuron on a mug-shaped branched manifold}

\author{
Mohamed Gheouali \\ University of Sciences and Technologies Houari Boumediene (USTHB), Mathematical Faculty, 16111 El \\ Alia, Bab Ezzouar, Algeria. \\ mohamegheouali@gmail.com \\ Tounsia Benzekri \\ University of Sciences and Technologies Houari Boumediene (USTHB), Mathematical Faculty, $16111 \mathrm{El}$ \\ Alia, Bab Ezzouar, Algeria. \\ tbenzekri@usthb.dz \\ René Lozi \\ University of Nice Sophia-Antipolis, U.C.A., UMR CNRS 7351, Nice, France \\ Rene.LOZI@univ-cotedazur.fr ${ }^{*}$ \\ Guanrong Chen \\ Department of Electrical Engineering, City University of Hong Kong, Hong Kong SAR, China \\ gchen@ee.cityu.edu.h
}

Received (to be inserted by publisher)

\begin{abstract}
Based on the Hodgkin-Huxley and Hindmarsh-Rose models, this paper proposes a geometric phenomenological model of bursting neuron in its simplest form, describing the dynamic motion on a mug-shaped branched manifold, which is a cylinder tied to a ribbon. Rigorous mathematical analysis is performed on the nature of the bursting neuron solutions: the number of spikes in a burst, the periodicity or chaoticity of the bursts, etc. The model is then generalized to obtain mixing burst of any number of spikes. Finally, an example is presented to verify the theoretical results.
\end{abstract}

Keywords: Bursting oscillation; Spike, Poincaré map, Horseshoe attractor, Chaos.

\section{Introduction}

In the human brain, which is believed to be the most complex of all biological systems, there are more that 100 billion of biological neurons. Each neuron is connected to many others (up to 10,000) via synapses in intricate patterns [Stiles \& Jernigan, 2010]. Neuronal signals consist of short electrical pulses, which can be observed by placing a fine electrode close to the soma or axon of a neuron. These neurons may present spiking and bursting activities. Since all spikes of a given neuron look alike, the form of the action potential does not carry much information. Rather, it is the number and the timing of spikes that matter. However, because spiking-bursting activities result from higher-dimensional nonlinear dynamics of various

*rlozi@unice.fr 

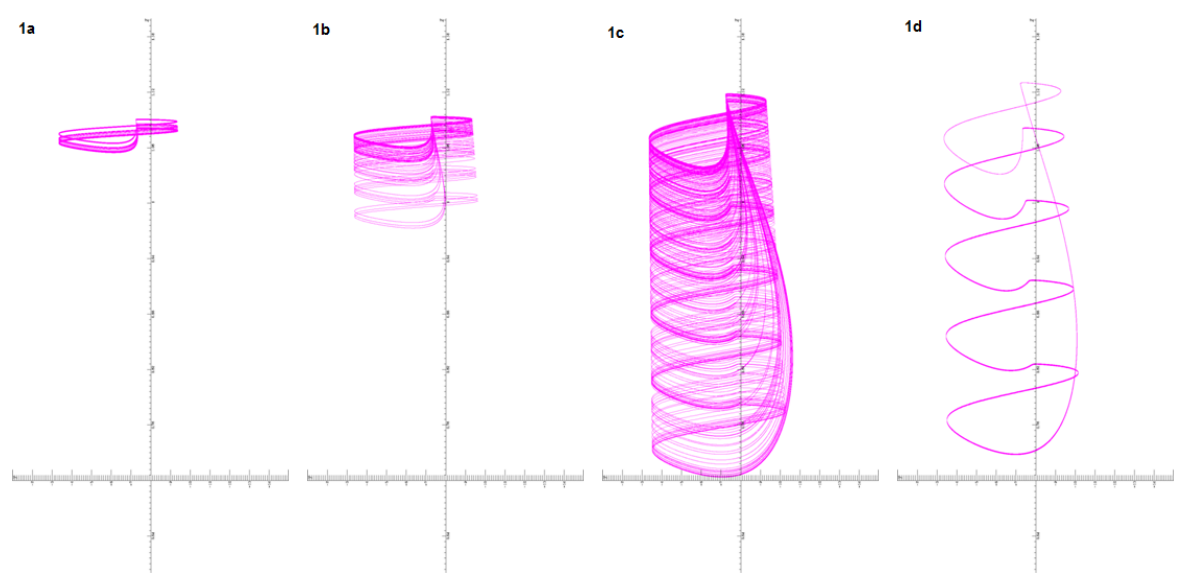

Figure 1. Solution of the Hindmarsh-Rose equation on the $(y, z)$ plane for $a=1, b=3, c=-3, d=5, s=4, I=5, x_{1}=-1.03$. Initial values $x_{0}=0, y_{0}=2.22, z_{0}=1$ and different values of $\epsilon: 1 a, \epsilon=0.0013,1 b, \epsilon=0.0165,1 c, \epsilon=0.04,1 d, \epsilon=0.05$.

ionic currents flowing through the membrane channels, it is very difficult to model exactly all the biochemical reactions that are interacting in a single neuron.

Since the pioneering works of Hodgkin and Huxley [Hodgkin \& Huxley, 1952] (the H-H model), which studied the squid giant axon, several channel-based models composing of several nonlinear equations have been designed to capture the physiological processes in the membrane (see [Shilnikov \& Rulkov, 2003; Shilnikov \& Kolomiets, 2008] for a survey). Contrarily, phenomenological models try only to replicate the characteristic features of the bursting behavior without direct relating to what happens in the neuron from a biological perspective. Among them, the most known is the Hindmarsh-Rose model [Corson, 2009] (the H-R model). In their first paper dated 1982, Hindmarsh and Rose introduced a model of two ordinary differential equations (ODE), which comes from a modification of the Fitzhugh-Nagumo system derived from a two-equation reduction of the H-H model of four ODE [Hindmarsh \& Rose, 1982]. Two years later in [Hindmarsh \& Rose, 1984], they expanded their simple 2-D into a more sophisticated 3-D model, which demonstrates almost all types of robust activities in the $\mathrm{H}-\mathrm{H}$ model:

$$
\left\{\begin{array}{l}
\dot{x}(t)=y(t)-a x^{3}(t)+b x^{2}(t)+I-z(t) \\
\dot{y}(t)=c-d x^{2}(t)-y(t) \\
\dot{z}(t)=\epsilon\left(s\left(x(t)-x_{1}\right)-z(t)\right) .
\end{array}\right.
$$

In this model, the voltage across the neuron's membrane is represented by the variable $x$, meanwhile the other two variables $y$ and $z$ describe some ionic currents' kinetics. Because the parameter $\epsilon$ is small, system (1) is called a slow-fast system. Both H-H and H-R models have been thoroughly and extensively studied using dynamic systems theory and tools. However, due to the innermost nonlinearities, system (1) is not easy for strictly mathematical proofs of solution properties. Moreover, small change in one of the seven parameters, $a, b, c, d, I, \epsilon, x_{1}$, can lead to very different solutions (see Fig. 1).

Aside from the H-R model, the FitzHugh-Nagumo (FHN) system, modeled by a two-dimensional nonlinear differential equation, when used with fractional derivative (Fractional FitzHugh-Nagumo FFHN), exhibits Mixed-Mode Oscillations (MMO) similar to the burst of spikes of the H-H model [Abdelouahab et al., 2019].

In what follows, we introduce a geometric phenomenological model of bursting neuron, which has some similarities with the H-R model. This model, which defines motion on a mug-shaped branched manifold, is presented in its simplest form where the branched manifold is simply a cylinder tied to a ribbon. This model belongs to a family of geometrical models for which the cylinder can be replaced by a cone or a paraboloid and the single ribbon can be duplicated. This geometrical mug-shaped model allows some rigorous mathematical analysis on the nature of its solutions: the number of spikes in a burst, the periodicity or chaoticity of the bursts, etc.

Next, in Sec. 2, the simplest geometric mug-shaped model on a branched manifold is defined. This 


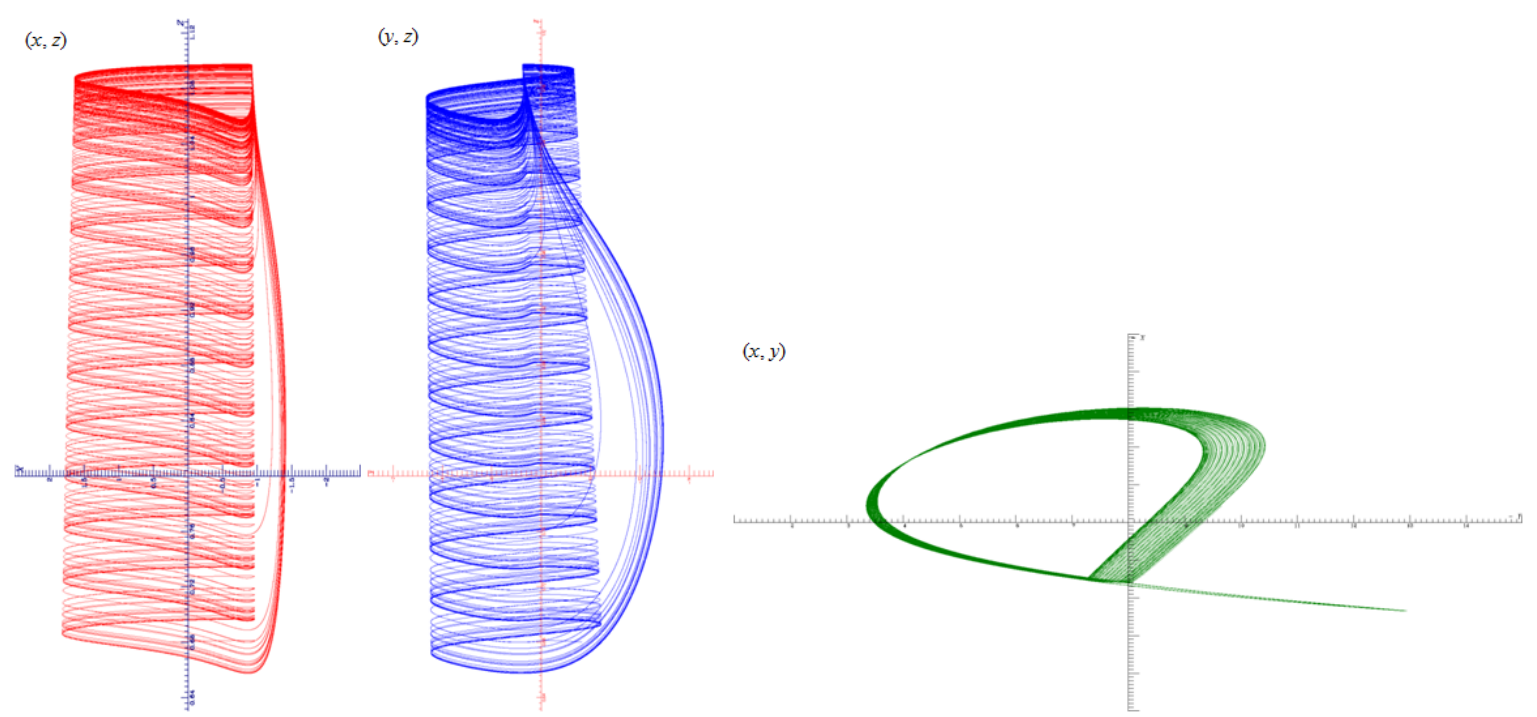

Figure 2. Solution of the Hindmarsh-Rose equation on the $(x, z),(y, z),(x, y)$ planes for $a=1, b=3, c=-3, d=5, s=4, I=$ $5, x_{1}=-1.03, \epsilon=0.002$. Initial values $x_{0}=0, y_{0}=2.22, z_{0}=1$.

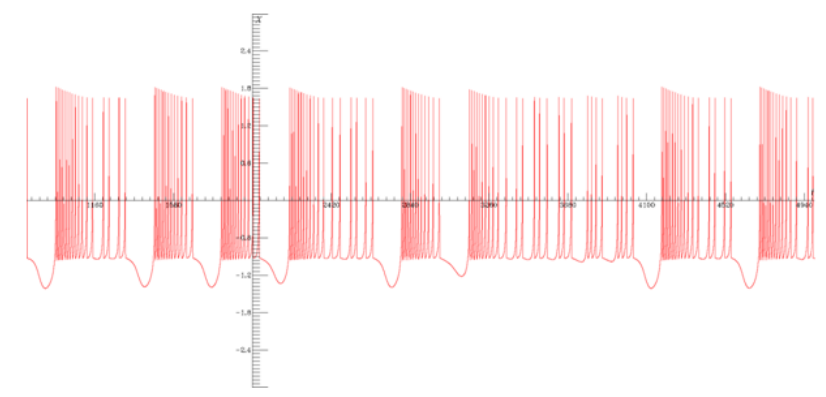

Figure 3. Solution of the Hindmarsh-Rose equation on the $(x, t)$, coordinates for $a=1, b=3, c=-3, d=5, s=4, I=$ $5, x_{1}=-1.03, \epsilon=0.002$. Initial values $x_{0}=0, y_{0}=2.22, z_{0}=1$.

simplest model generates only bursting orbits, which mix burst of $n$ and $n+1$ spikes. In Sec. 3, properties of periodic and quasiperiodic orbits are studied, and rigorous proofs of their existence are given. In Sec. 4, a more complex case of the model is studied, which generates chaotic bursting orbits with burst of $n$ and $n+1$ spikes. In Sec. 5, the model is generalized to obtain mixing burst of any number of spikes. An example with bursts of 4 to 8 spikes is discussed. Finally, in Sec. 6, a brief conclusion is drawn.

\section{Geometrical model of neuron on a mug-shaped branched manifold: simplest case}

In this section, a new model of neuron defined on manifold is introduced, inspired the shape of solutions of the H-R model. Fig. 2 shows a solution of the Hindmarsh-Rose equation on the $(x, z),(y, z)$, and $(x, y)$ planes. Figure 3 displays the $x$ signal versus time, highlighting the spiking-bursting phenomenon.

Then, a mug-shaped branched manifold is introduced, consisting of a cylinder of finite length connected to a ribbon defined using a sheaf of rectangular part on the plane (Fig. 4). The geometrical model is defined by curves circling upward around this cylinder, and jumping back to its basis through the ribbon, when they cross a threshold on the upper part of the cylinder (Fig. 5).

Formally, the dynamics on the mug-shaped manifold is composed of two folds: the dynamics on the cylinder, and the dynamics on the ribbon. 

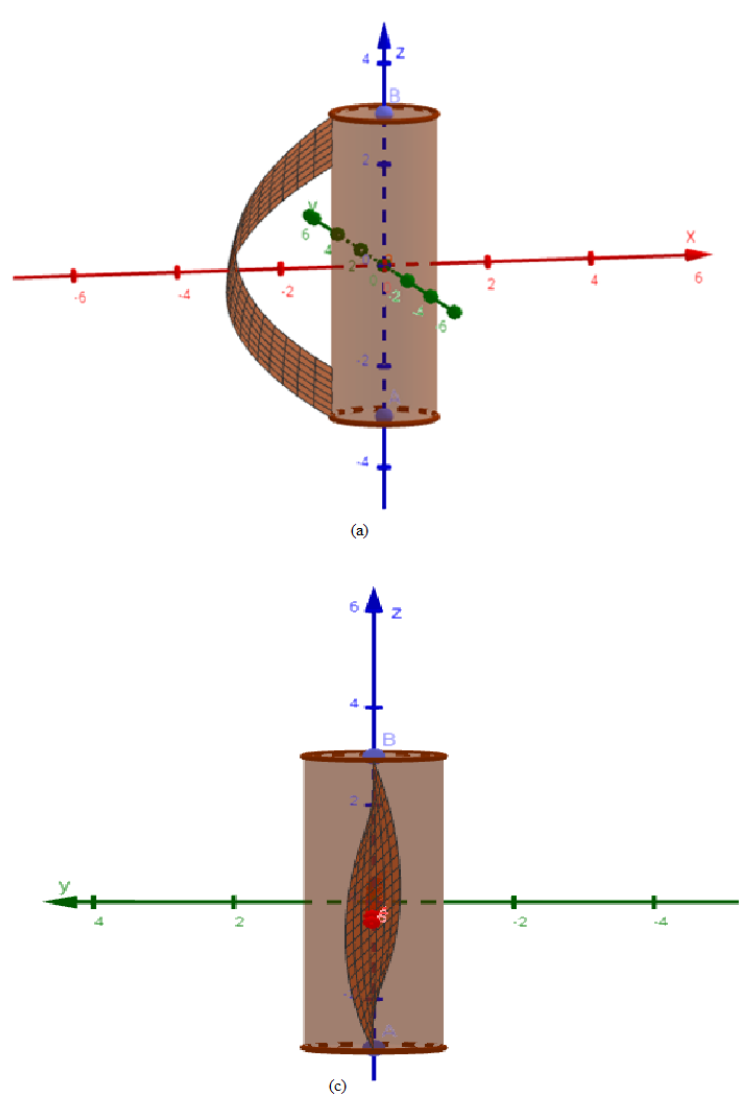
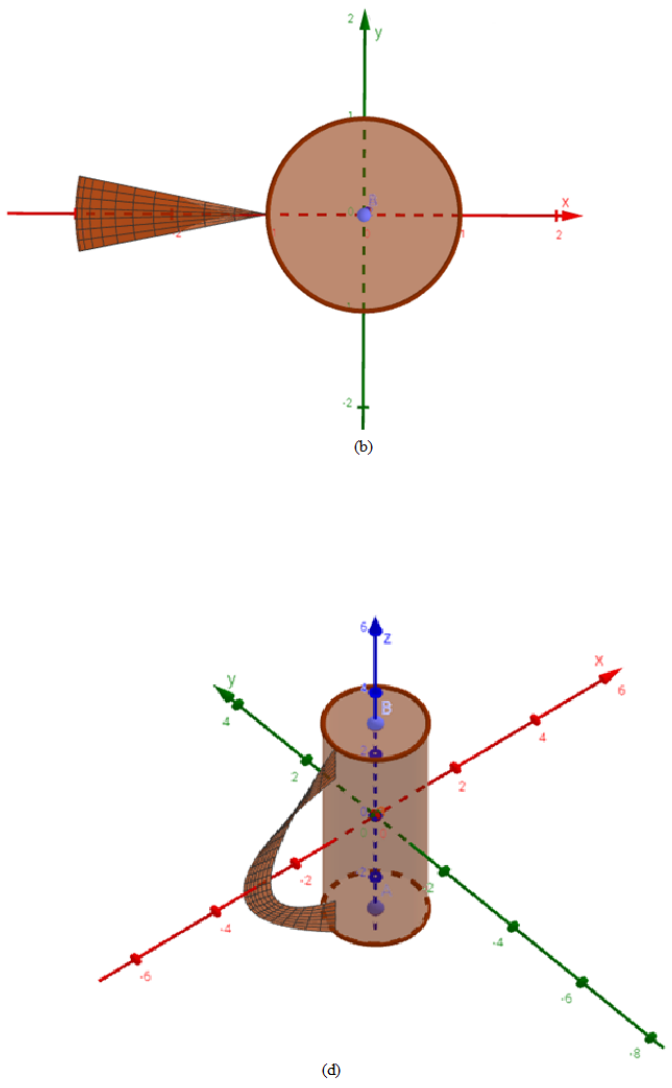

Figure 4. Ribbon and cylinder view in perspective in coordinate $(O, x, y, z)$. (a) side view, (b) projection on the plane $(x, y)$, (c) projection on the plane $(y, z)$, (d) top view

\subsection{Dynamics on the cylinder}

Let $S$ of $\mathbb{R}^{3}$ be the cylindrical region defined by $x^{2}+y^{2}=1$ and $-s-1 \leqslant z \leq s+1$, where $s \in \mathbb{R}^{+}$is the half length of the cylinder.

We first define precisely what we call spike, burst and bursting trajectories (or bursting orbit, or bursting solution) of the geometrical model.

\subsubsection{Spike, burst and bursting orbits}

As shown in Fig. 3, a spike in the H-R model (1) is the projection of a complete revolution of the signal around the $z$ axis, on the $(x, t)$ coordinates. In the same manner, a spike in the geometrical model corresponds to the part of the trajectory turning around the cylinder.

A burst occurs when neuron activity alternates between a quiescent state and repetitive spiking as shown in Fig. 6 [Izhikevich, 2007]. In the proposed geometrical model, a burst is a piece of the trajectory starting from the lower part of the cylinder, circling many times around it, leaving the cylinder as soon as it can escape from it to the top part of the ribbon, and ending at the boundary between the low part of the ribbon and the cylinder.

A bursting orbit $\mathcal{B}\left(z_{0}\right)$ is a continuous trajectory encompassing an infinite sequence of bursts $\mathcal{B}\left(z_{0}\right)=$ $\left\{B_{0}\left(z_{0}\right), B_{1}\left(z_{0}\right), \ldots, B_{m}\left(z_{0}\right), \ldots\right\}$ defined by its initial point $\left(-1,0, z_{0}\right)$ at the initial time $t_{0}, z_{0}=z\left(t_{0}\right)$.

It can be periodic, pseudo-periodic or chaotic.

Now, assume that for the burst $B_{m_{\left(z_{0}\right)}}$, the trajectory starts to turn around the cylinder at the time $\tau_{m_{\left(z_{0}\right)}}^{c}$ and the point $\left(-1,0, z\left(\tau_{m_{\left(z_{0}\right)}}^{c}\right)\right)$, with $-s-1 \leq z\left(\tau_{m_{\left(z_{0}\right)}}^{c}\right)<-s$, and leaves the cylinder $S$ at the time 


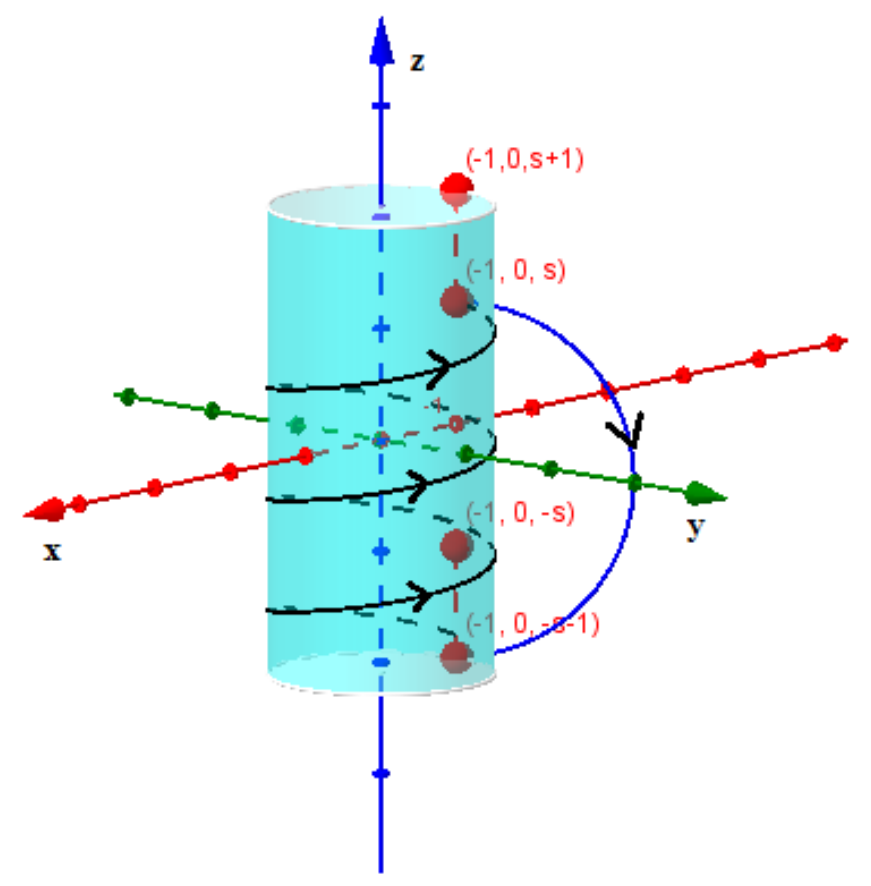

Figure 5. Geometrical representation of dynamics of a neuron on the cylinder $S$

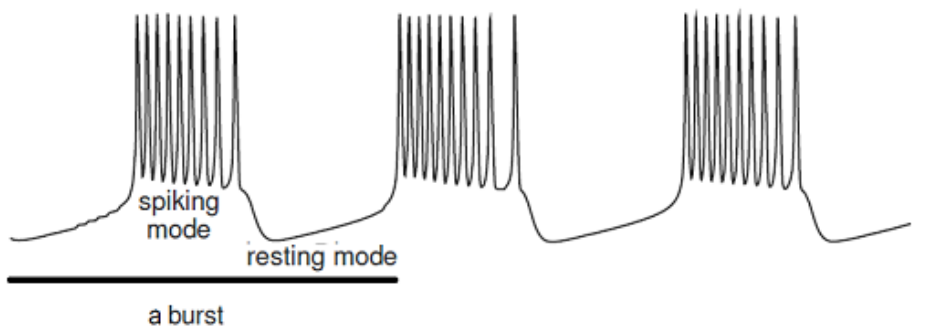

Figure 6. Bursting trajectory with three bursts [Izhikevich, 2007].

$\tau_{m_{\left(z_{0}\right)}}^{r}$ and the point $\left(1,0, z\left(\tau_{m_{\left(z_{0}\right)}}^{r}\right)\right)$, with $s \leq z\left(\tau_{m_{\left(z_{0}\right)}}^{r}\right)<s+1$, to traverse on the ribbon.

The point $\left(-1,0, z\left(\tau_{m_{\left(z_{0}\right)}}^{c}\right)\right)$ for $m>1$ is defined as the end of the trajectory of $B_{m-1_{\left(z_{0}\right)}}$, which in turn is defined by $B_{m-2_{\left(z_{0}\right)}}$ and recursively by $B_{m-3_{\left(z_{0}\right)}}, \ldots, B_{2_{\left(z_{0}\right)}}, B_{1_{\left(z_{0}\right)}}$ and by $\tau_{0_{\left(z_{0}\right)}^{c}}^{c}=t_{0}$ for $B_{0_{\left(z_{0}\right)}}$.

For simplicity, in the case where no ambiguity can occur, denote $\tau_{m_{\left(z_{0}\right)}}^{r}\left(\right.$ resp. $\left.\tau_{m_{\left(z_{0}\right)}^{c}}^{c}\right)$ by $\tau_{m}^{r}$, (resp. $\left.\tau_{m}^{c}\right)$ and $B_{m_{\left(z_{0}\right)}}$ by $B_{m}$.

The duration of this burst is equal to $\tau_{m+1}^{c}-\tau_{m}^{c}$ i.e.

$$
t \in T_{m}^{B}=\left[\tau_{m}^{c}, \tau_{m+1}^{c}\left[=T_{m}^{B c y l} \cup T_{m}^{B r i b}=\left[\tau_{m}^{c}, \tau_{m}^{r}\left[\cup \left[\tau_{m}^{r}, \tau_{m+1}^{c}[.\right.\right.\right.\right.\right.
$$


The number of spikes within a burst is the total number of turns that the bursting orbit did around the cylinder $S$.

\subsubsection{Intervals of injection and reinjection}

Here, consider a trajectory which arrives from the ribbon and begins to turn around the cylinder.

Definition 2.1. Interval of reinjection

Let $I_{s}^{c}=\left[-s-1,-s\left[\right.\right.$ be an interval of the $z$-axis, and $\bar{I}_{s}^{c}=\{-1\} \times\{0\} \times I_{s}^{c}$ be the interval that contains all possible initial points of trajectories of the geometrical model, leaving the ribbon to turn around the cylinder. This interval is called the interval of reinjection.

Definition 2.2. Interval of injection

Let $I_{s}^{r}=\left[s, s+1\left[\right.\right.$ be an interval of the $z$-axis, and $\bar{I}_{s}^{r}=\{-1\} \times\{0\} \times I_{s}^{r}$ be the interval that contains all possible final points of trajectories of the geometrical model, leaving the cylinder to move to the ribbon. This interval is called the interval of injection.

For simplicity, in the rest of this article, we identify the point $(-1,0, z) \in\{-1\} \times\{0\} \times[-s-1,-s[$ as the point $z \in[-s-1,-s$ [ of the $z$-axis and the point $(-1,0, z) \in\{-1\} \times\{0\} \times[s, s+1[$ as the point $z \in\left[s, s+1\left[\right.\right.$ of the $z$-axis, see Fig. 5 . Consequently, we identify the interval $\bar{I}_{s}^{c}$ (resp. $\bar{I}_{s}^{r}$ ) with the interval $I_{s}^{c}\left(\operatorname{resp} . I_{s}^{r}\right)$.

\subsubsection{Equation of trajectories on the cylinder}

In order to define, in a simplified manner, the equations of the motion belonging to the cylinder, in every burst $B_{m}$, use the local time $\theta$ :

$$
\forall t \in T_{m}^{B c y l}, \theta=t-\tau_{m}^{c} .
$$

For $\theta \in\left[0, \tau_{m}^{r}-\tau_{m}^{c}\right.$ [, equations of motion are given by the following differential equations:

$$
\left\{\begin{array}{l}
\ddot{x}(\theta)=-4 \pi^{2} x(\theta), \\
\ddot{y}(\theta)=-4 \pi^{2} y(\theta), \\
\dot{z}(\theta)=1 .
\end{array}\right.
$$

Then, in the case where the spikes are regularly spaced, at each burst $B_{m}$ the solution on the cylinder is given by

$$
\left\{\begin{array}{l}
x(\theta)=\cos (2 \pi \theta+\pi), \\
y(\theta)=\sin (2 \pi \theta+\pi), \\
z(\theta)=\theta+z_{m}^{c} .
\end{array}\right.
$$

The initial condition of the solution in $B_{m}$ verifies $z_{m}^{c} \in I_{s}^{c}$. During the burst, this solution is turning around the cylinder, as long as $z(\theta)<s$, until $x(\theta) \neq-1$.

When $z(\theta) \geqslant s$ and $x(\theta)=-1$, the trajectory leaves the cylinder and traverses on the ribbon.

\subsubsection{The half-Poincaré map on the cylinder}

In this article, consider only the case where spikes are regularly spaced versus time at the same burst. This is due to the third equation of $(3), \dot{z}(\theta)=1$. However, it is easy to relax this constraint, allowing more flexibility to the behavior of the spike, by modifying slightly this equation as $\dot{z}(\theta)=f(\theta)$, with for example $f(\theta)=\theta+\frac{1}{2}$.

At any burst, the trajectory starting from interval $\bar{I}_{s}^{c}$ must eventually exit from $S$ through $\bar{I}_{s}^{r}$. Since the interval $\bar{I}_{s}^{c}\left(\right.$ resp. $\left.\bar{I}_{s}^{r}\right)$ is identified with the interval $I_{s}^{c}\left(\right.$ resp. $\left.I_{s}^{r}\right)$, one can define a map $\Psi_{1}: I_{s}^{c} \longrightarrow I_{s}^{r}$ 
following trajectories that start from $I_{s}^{c}$ until they first meet $I_{s}^{r}: \Psi_{1}\left(z_{m}^{c}\right)=z\left(\tau_{m}^{r}\right)=z_{m}^{r}$. For all $z_{m}^{c} \in I_{s}^{c}$, there is $\theta^{*}=k, k \in \mathbb{N}$, such that $\Psi_{1}\left(z_{m}^{c}\right)=k+z_{m}^{c}$, for which one has

$$
s \leq k+z_{m}^{c}<s+1
$$

because $x\left(\theta^{*}\right)=-1$ and $y\left(\theta^{*}\right)=0$. By writing $2 s$ as $2 s=[2 s]+\alpha, 0 \leq \alpha<1$, one has

$$
[2 s]+\left(-z_{m}^{c}-s+\alpha\right) \leq k<[2 s]+\left(-z_{m}^{c}-s+\alpha\right)+1 \text {. }
$$

From the fact that

$$
\left\{\begin{array}{l}
1 \leq\left(-z_{m}^{c}-s+\alpha\right)<2 \quad \text { for } \quad z_{m}^{c} \in[-s-1,-s-1+\alpha[ \\
0<\left(-z_{m}^{c}-s+\alpha\right) \leq 1 \quad \text { for } \quad z_{m}^{c} \in[-s-1+\alpha,-s[
\end{array}\right.
$$

it follows that

$$
k=\left\{\begin{array}{lll}
{[2 s]+2} & \text { for } & z_{m}^{c} \in[-s-1,-s-1+\alpha[ \\
{[2 s]+1} & \text { for } \quad z_{m}^{c} \in[-s-1+\alpha,-s[
\end{array}\right.
$$

Therefore, one has $\Psi_{1}: I_{s}^{c} \longrightarrow I_{s}^{r}$ defined by

$$
\Psi_{1}(z)=\left\{\begin{array}{lll}
z+[2 s]+2 & \text { for } & z \in[-s-1,-s-1+\alpha[ \\
z+[2 s]+1 & \text { for } & z \in[-s-1+\alpha,-s[.
\end{array}\right.
$$

(See example with $s=2.2$, shown in Fig. 7).

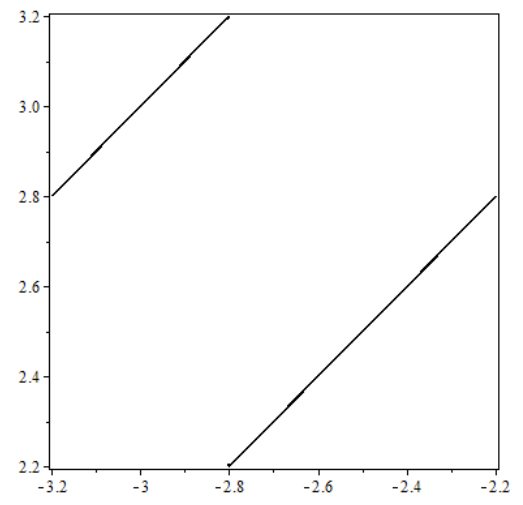

Figure 7. The graph of the map $\Psi_{1}$ for $s=2.2$

At a burst, as will be seen in Section 3, this map can define patterns of $[2 s]+1$ and $[2 s]+2$ spikes.

This half-Poincaré map is coupled with a second half-Poincaré map on the ribbon linking the interval of injection to the interval of reinjection.

\subsection{Dynamics on the ribbon}

When $x=-1$ and $z \geq s$, the orbits leave the cylinder $S$ towards the plane $(x, z)$ to form the phase of quiescence and then return to the cylinder.

Here, assume that the duration between bursts (interbursts or interspikes), representing the phase of quiescence, is the same for all bursts and is equal to $2 T$.

$$
T_{m}^{B r i b}=\left[\tau_{m}^{r}, \tau_{m+1}^{c}\left[=\left[\tau_{m}^{r}, \tau_{m}^{r}+2 T[.\right.\right.\right.
$$




\subsubsection{Ribbon defined by a sheaf of rectangular part of plane}

On the ribbon, just like on the cylinder, define in every burst $B_{m}$ the local time

$$
\theta: \forall t \in T_{m}^{B r i b}, \theta=t-\tau_{m}^{r} \in[0,2 T[.
$$

Also, assume that the projection on the $z$-axis of every trajectory on the ribbon has the same length $2 s+1$, and has a parabolic shape. Due to the Poincaré-Bendixon theorem, which implies that on a plane no trajectory of a dynamical system can cross another trajectory, the ribbon cannot belong to a single plane. In order to avoid such inappropriate crossing, define each identical parabola-shaped trajectory on a separate half-plane $H_{m}$, formed by the angle $\hat{\mu}_{m}$ between $H_{m}$ and the half-plane $H=\left\{X \geq 0,(Y, Z) \in \mathbb{R}^{2}\right\}$, for which both systems of coordinates $(O, x, y, z)$ and $(\tilde{O}, X, Y, Z)$ are defined by

$$
\left\{\begin{array}{l}
X=x+1 \\
Y=y \\
Z=z
\end{array}\right.
$$

and $\tilde{O}=(-1,0,0)$ in the system $(O, x, y, z)$, together with the family of systems of coordinates $\left(\tilde{O}, \tilde{X}_{m}, \tilde{Y}_{m}, \tilde{Z}_{m}\right)$ defined by

$$
\left(\begin{array}{c}
\tilde{X}_{m} \\
\tilde{Y}_{m} \\
\tilde{Z}_{m}
\end{array}\right)=\left(\begin{array}{ccc}
\cos \left(\hat{\mu}_{m}\right) & \sin \left(\hat{\mu}_{m}\right) & 0 \\
-\sin \left(\hat{\mu}_{m}\right) & \cos \left(\hat{\mu}_{m}\right) & 0 \\
0 & 0 & 1
\end{array}\right)\left(\begin{array}{l}
X \\
Y \\
Z
\end{array}\right)
$$

(Fig. 8) each of which helps to define the half-plane $H_{m}$ (Fig. 9), as follows:

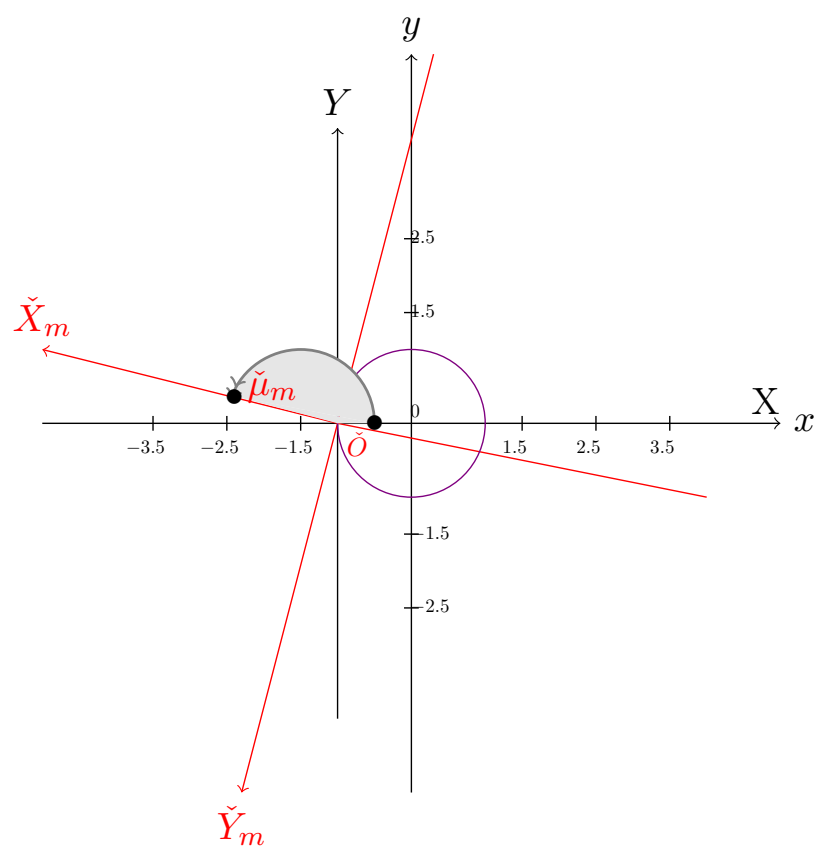

Figure 8. Systems of coordinates used to define the half-plane $H_{m}$

$$
H_{m}=\left\{\tilde{X}_{m} \geq 0, \tilde{Y}_{m}=0, \tilde{Z}_{m} \in \mathbb{R}\right\}
$$


On each half-plane $H_{m}$, the parabolic trajectory is parametrized by $\theta$ as

$$
\left\{\begin{array}{l}
\tilde{X}_{m}(\theta)=\beta_{0} \theta^{2}+\gamma_{0} \theta+\delta_{0} \\
\tilde{Z}_{m}(\theta)=\beta_{1} \theta+\gamma_{1}
\end{array}\right.
$$

and verifies $\tilde{Z}_{m}(0)=z_{m}^{r}, \tilde{Z}_{m}(2 T)=z_{m}^{r}-(2 s+1)$ for the $z$-coordinate, and

$$
\left\{\begin{array}{l}
\tilde{X}_{m}(0)=0, \\
\tilde{X}_{m}(T)=M, \\
\tilde{X}_{m}(2 T)=0,
\end{array}\right.
$$

for the $x$-coordinate (Fig. 9), where $M$ is a constant defining the maximal amplitude of the parabola. Under those conditions, it is straightforward to obtain

$$
\begin{gathered}
\beta_{0}=-\frac{M}{T^{2}}, \quad \gamma_{0}=\frac{2 M}{T}, \quad \delta_{0}=0 \quad \text { and } \quad \beta_{1}=\frac{-(2 s+1)}{2 T}, \quad \gamma_{1}=z_{m}^{r}, \\
\left\{\begin{array}{l}
\tilde{X}_{m}(\theta)=\frac{M \theta}{T}\left(2-\frac{\theta}{T}\right), \\
\tilde{Z}_{m}(\theta)=\frac{-(2 s+1)}{2 T} \theta+z_{m}^{r} .
\end{array}\right.
\end{gathered}
$$

Of course, the angle $\hat{\mu}_{m}$ must be bounded, for all the values of $m$. One can choose for example $\hat{\mu}_{m}=$ $\frac{\pi}{8}\left(z_{m}^{r}-s\right)+\frac{15 \pi}{16}$, which satisfies $\frac{15 \pi}{16}<\hat{\mu}_{m}<\frac{17 \pi}{16}$. The ribbon plotted in (Fig. 9) is defined with this value of $\hat{\mu}_{m}$, and $T=1, s=2, M=2$.

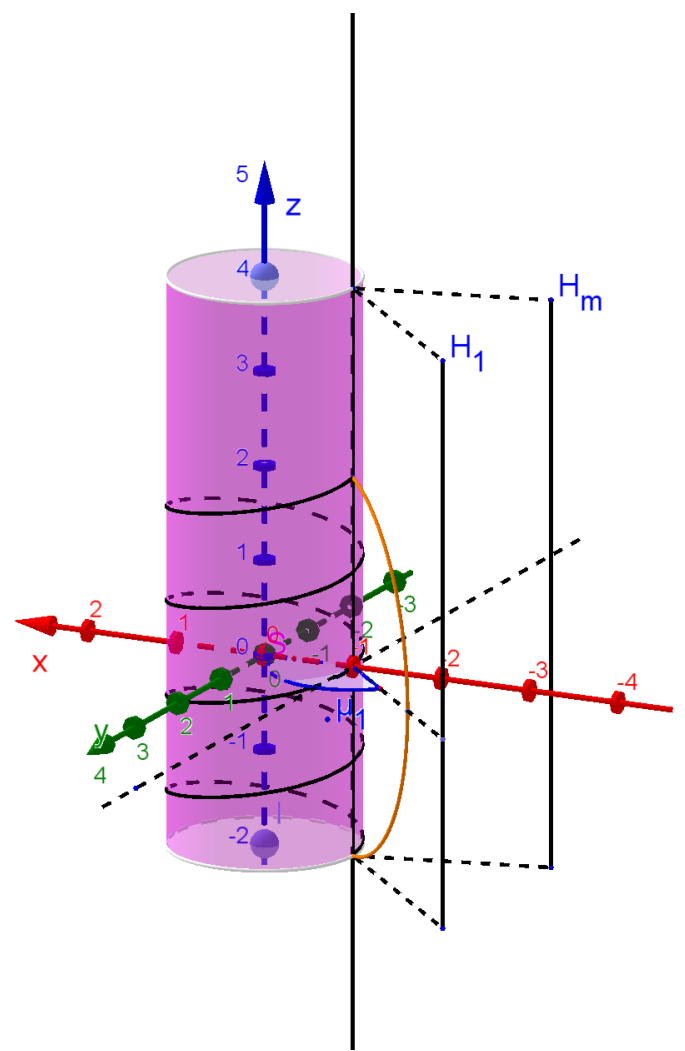

Figure 9. The dynamics of a neuron on the cylinder giving spiking and on the projection on $H_{1}$ giving the first period of quiescence, where the family $\left\{H_{m}\right\}_{m=0, \infty}$ is a sheaf of plane. 


\subsubsection{The half-Poincaré map on the ribbon}

This second half-Poincaré map is defined, following the solution that starts from the reinjection interval until it reaches the injection interval.

Let $z_{m}^{r} \in I_{s}^{r}$. Considering $z(s)=\left(-2 s-1+z_{m}^{r}\right) \in I_{s}^{c}$, set $z_{m+1}^{c}=\left(-2 s-1+z_{m}^{r}\right)$. Then, define the $\operatorname{map} \Psi_{2}: I_{s}^{r} \longrightarrow I_{s}^{c}$ by $\Psi_{2}\left(z_{m}^{r}\right)=z\left(\tau_{m+1}^{c}\right)=z_{m+1}^{c}$, and

$$
\Psi_{2}(z)=z-(2 s+1) .
$$

\subsubsection{Poincaré return map for a neuron}

The composition of both half-Poincaré maps $\Psi_{1}$ and $\Psi_{2}$ defines a Poincaré map $\Phi=\Psi_{2} \circ \Psi_{1}$ on the reinjection interval. The map $\Psi_{1}$ is defined on $I_{s}^{c}$ and takes values in $I_{s}^{r}$, and $\Psi_{2}$ takes values in $I_{s}^{c}$. Now, one has $\Phi_{s}: I_{s}^{c} \longrightarrow I_{s}^{c}$, where $\Phi_{s}=\Psi_{2} \circ \Psi_{1}$ is given by

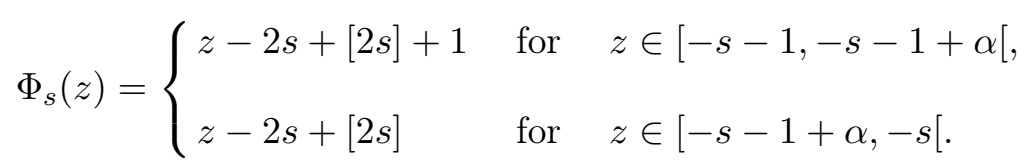

By the change of coordinates, $\zeta=z+s+1, \Phi(\zeta)=\Phi_{s}(z)+s+1$, the map $\Phi_{s}$ is isomorphic to the map $\Phi:[0,1[\rightarrow[0,1[$, which is defined by

$$
\Phi(\zeta)= \begin{cases}\zeta+1-\alpha & \text { for } \quad \zeta \in I_{0}, \\ \zeta-\alpha & \text { for } \quad \zeta \in I_{1},\end{cases}
$$

with $\alpha=2 s-[2 s], I_{0}=\left[0, \alpha\left[\right.\right.$, and $I_{1}=[\alpha, 1[$.

Let $\zeta_{0} \in\left[0,1\left[\right.\right.$, and set $\zeta_{0}=\Phi^{0}\left(\zeta_{0}\right)$ and $\zeta_{m}=\Phi^{m}\left(\zeta_{0}\right) \in\left[0,1\left[\right.\right.$, for $m \geq 1$. The point $\Phi^{m}\left(\zeta_{0}\right)-(s+1)$ denotes the position where the bursting orbit begins to turn around the cylinder to form the burst $B_{m_{\left(z_{0}\right)}}$ with $z_{0}=\Phi^{0}\left(\zeta_{0}\right)-(s+1)$.

It is equivalent to consider the bursting orbit $\mathcal{B}\left(z_{0}\right)=\left\{B_{0}\left(z_{0}\right), B_{1}\left(z_{0}\right), \ldots, B_{m}\left(z_{0}\right), \ldots\right\}$ or the bursting orbit of the Poincaré map $\Phi\left(\zeta_{0}\right)=\left\{\Phi^{0}\left(\zeta_{0}\right), \Phi^{1}\left(\zeta_{0}\right), \ldots, \Phi^{m}\left(\zeta_{0}\right), \ldots\right\}$.

From equation (5), it can be seen that the number of spikes within the burst $B_{m}$ is $([2 s]+2)$ if $\Phi^{m}\left(\zeta_{0}\right)$ belongs to $I_{0}$ and $([2 s]+1)$ if $\Phi^{m}\left(\zeta_{0}\right)$ belongs to $I_{1}$.

An example of the distribution of spikes and quiescence regimes at a burst is illustrated by Fig. 10 .

\section{Periodic and quasi-periodic bursting orbits}

In this section, the focus is on the number of spikes of each burst of a periodic bursting orbit.

Depending on the rationality or irrationality of the parameter $\alpha$, the shape of the burst in such an orbit is quite different. There exist only periodic orbits for rational $\alpha$, instead for irrational $\alpha$, the model can have quasiperiodic orbits.

\subsection{Condition of existence of periodic bursting orbit}

Proposition 1. If $\alpha$ is a rational number, $\alpha=\frac{p}{q}$, with $p, q \in N^{*}, 0<p<q$ and $\operatorname{pgcd}(p, q)=1$, then for any initial value $\zeta_{0} \in\left[0,1\left[\right.\right.$, the bursting orbit $\Phi\left(\zeta_{0}\right)$ is periodic of period $q$ (i.e. $\left.\Phi^{q}\left(\zeta_{0}\right)=\zeta_{0}\right)$; moreover, $q$ is the smallest possible period.

Proof. For any initial condition $\zeta \in[0,1[$, rewrite the map $\Phi$ as

$$
\Phi(\zeta)=\zeta+\gamma \quad \bmod 1
$$




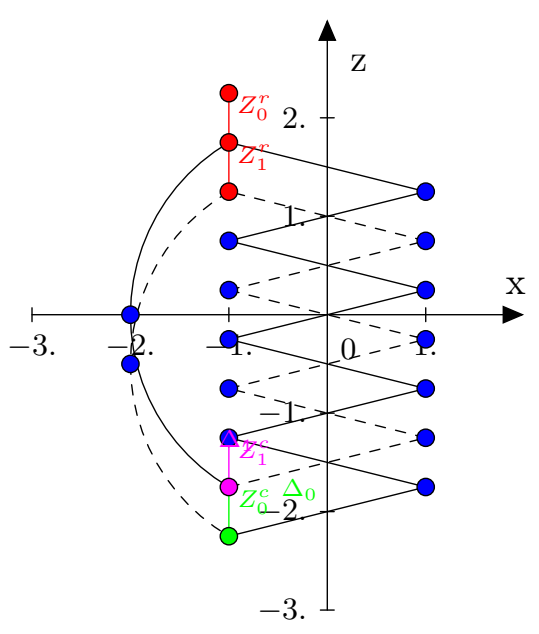

Figure 10. Plot of a bursting for $\alpha=\frac{1}{2}$

where $\gamma=1-\alpha$. Then, $\Phi^{q}(\zeta)=\zeta+q\left(1-\frac{p}{q}\right)=\zeta+(q-p),(\bmod 1)$, hence $\Phi^{q}(\zeta)=\zeta$.

If $q$ is not the smallest period, there exists a smallest period $\tilde{q}$ with $\tilde{q}<q$ and $q=n \tilde{q}+r$, where $r<\tilde{q}$. Then, $\zeta=\Phi^{q}(\zeta)=\Phi^{n \tilde{q}+r}(\zeta)=\Phi^{r}\left(\Phi^{n \tilde{q}}\right)(\zeta)=\Phi^{r}(\zeta)$, which is impossible.

Remark 3.1. This is equivalent to that the corresponding orbit $\mathcal{B}\left(z_{0}\right)$ is periodic with $q$ bursts belonging to the period.

Remark 3.2. It is important to point out the difference between the period of the bursting orbit $\Phi\left(\zeta_{0}\right)$ of the Poincare map, which is an integer, and the period of the bursting orbit of the geometrical HR model, which is a duration $T_{s}$. In fact, even if $q$ bursts are belonging to the period of the bursting orbit $\mathcal{B}\left(z_{0}\right)$, their periods can be different, depending on the value of $\alpha=\frac{p}{q}$. It will be proved bellow in Theorem 1 that $T_{s}=(2 T q+p([2 s]+2)+(q-p)([2 s]+1))$.

Remark 3.3. Two cylinders of nearly equal lengths can generate bursting orbits with a very different periods. As an example, for $s_{1}=1+\frac{3}{5}$, the period is 5 while for $s_{2}=1+\frac{3001}{5000}$ the period is 5000 .

\subsection{Distribution of spikes and bursts in a periodic bursting orbit}

\subsubsection{Number of spikes and bursts}

Theorem 1. For $\alpha=\frac{p}{q}$, a periodic bursting orbit with $q$ bursts has $p$ bursts of $([2 s]+2)$ spikes and $(q-p)$ bursts of $([2 s]+1)$ spikes.

Therefore, the period of such orbit is equal to $T_{s}=(2 T q+p([2 s]+2)+(q-p)([2 s]+1))$.

In order to prove this theorem, define a partition of the interval $I$ and prepare a lemma.

First, define $\triangle_{j}=\left[\frac{j}{q}, \frac{j+1}{q}\left[\subset I=\left[0,1\left[, 0 \leq j \leq q-1\right.\right.\right.\right.$. Thus, one can write $I_{0}=\bigcup_{j=0}^{p-1} \triangle_{j}$ and $I_{1}=\bigcup_{j=p}^{q-1} \triangle_{j}$. The intervals $\triangle_{j}$ form a partition of $I$ and

$$
\Phi\left(\triangle_{j}\right)=\left\{\begin{array}{l}
\triangle_{j+(q-p)} \text { when } \triangle_{j} \subset I_{0}(j \leq p-1), \\
\triangle_{j-p} \text { when } \triangle_{j} \subset I_{1}(j \geq p) .
\end{array}\right.
$$

\section{Lemma 1.}

- For $m_{1} \neq m_{2}, 0 \leq m_{i} \leq q-1, i=1,2$,

$$
\Phi^{m_{1}}\left(\triangle_{j}\right) \cap \Phi^{m_{2}}\left(\triangle_{j}\right)=\emptyset \text { for all } j, 0 \leq j \leq q-1 .
$$


- For $j_{1} \neq j_{2}, 0 \leq j_{1}, j_{2} \leq q-1$,

$$
\Phi^{m}\left(\triangle_{j_{1}}\right) \cap \Phi^{m}\left(\triangle_{j_{2}}\right)=\emptyset \text { for all } m
$$

Proof. [Proof of Lemma 1]

Suppose there exist $m_{1} \leq q-1$ and $m_{2} \leq q-1, m_{1}<m_{2}$ such that $\Phi^{m_{1}}\left(\triangle_{j}\right) \cap \Phi^{m_{2}}\left(\triangle_{j}\right) \neq \emptyset$. Then, there exists $\triangle_{l} \subset \bigcup_{j=0}^{q-1} \triangle_{j}$ such that $\Phi^{m_{1}}\left(\triangle_{j}\right)=\triangle_{l}$ and $\Phi^{m_{2}}\left(\triangle_{j}\right)=\Phi^{\left(m_{2}-m_{1}\right)}\left(\Phi^{m_{1}}\left(\triangle_{j}\right)\right)=\Phi^{\left(m_{2}-m_{1}\right)}\left(\triangle_{l}\right)=\triangle_{l}$.

Thus, each point $\zeta \in \triangle_{l}$ is periodic with period $\left(m_{2}-m_{1}\right)<q$. This contradicts the fact that $\Phi^{q}(\zeta)=\zeta$ for any $\zeta \in[0,1]$.

For the second case, it will be checked that if $j_{1} \neq j_{2}$, then $\Phi\left(\triangle_{j_{1}}\right) \cap \Phi\left(\triangle_{j_{2}}\right)=\emptyset$.

Indeed, for $j_{1} \neq j_{2}$, if $\triangle_{j_{1}}$ and $\triangle_{j_{2}}$ belong to $I_{0}$ then $\Phi\left(\triangle_{j_{i}}\right)=\triangle_{j_{i}}+(q-p)$ for $i=1,2$ and $j_{1}+(q-p) \neq j_{2}+(q-p)$.

One also has $\Phi\left(\triangle_{j_{i}}\right)=\triangle_{j_{i}}-p, i=1,2$ and $j_{1}-p \neq j_{2}-p$ if $\triangle_{j_{1}}$ and $\triangle_{j_{2}}$ belong to $I_{1}$ and so $\Phi\left(\triangle_{j_{1}}\right) \cap \Phi\left(\triangle_{j_{2}}\right)=\emptyset$.

In the case where $\triangle_{j_{1}} \subset I_{0}$ and $\triangle_{j_{2}} \subset I_{1}$, one has $\Phi\left(\triangle_{j_{1}}\right)=\triangle_{j_{1}}+(q-p)$ and $\Phi\left(\triangle_{j_{2}}\right)=\triangle_{j_{2}}-p$. Suppose that $\Phi\left(\triangle_{j_{1}}\right) \cap \Phi\left(\triangle_{j_{2}}\right) \neq \emptyset$. From (21), this implies that $\Phi\left(\triangle_{j_{1}}\right)=\Phi\left(\triangle_{j_{2}}\right)$, that is $\triangle_{j_{1}}+(q-p)=\triangle_{j_{2}}-p$, which is impossible since $j_{2} \leq q-1$.

The case of $\triangle_{j_{1}} \subset I_{1}$ and $\triangle_{j_{2}} \subset I_{0}$ is the same as this last one.

Therefore, for all $m, \Phi^{m}\left(\triangle_{j_{1}}\right) \cap \Phi^{m}\left(\triangle_{j_{2}}\right)=\emptyset$ if $j_{1} \neq j_{2}$.

Remark 3.4. Lemma 1 implies that

- For each $\triangle_{j} \subset \bigcup_{i=0}^{q-1} \triangle_{i},\left(\Phi^{0}\left(\triangle_{j}\right), \Phi^{1}\left(\triangle_{j}\right), \ldots, \Phi^{q-1}\left(\triangle_{j}\right)\right)$ are disjoint sets, so

$$
\bigcup_{m=0}^{q-1} \Phi^{m}\left(\triangle_{j}\right)=\bigcup_{j=0}^{q-1} \triangle_{j}=[0,1[.
$$

- Let $\zeta_{0} \in \triangle_{j}$. Then, $\left(\zeta_{0}, \Phi^{1}\left(\zeta_{0}\right), \ldots, \Phi^{q-1}\left(\zeta_{0}\right)\right) \in\left(\triangle_{j}, \Phi^{1}\left(\triangle_{j}\right), \ldots, \Phi^{q-1}\left(\triangle_{j}\right)\right)$, i.e. for $\zeta_{0} \in \triangle_{j}, \Phi^{m}\left(\zeta_{0}\right) \in$ $\Phi^{m}\left(\triangle_{j}\right)$, for $0 \leq m \leq q-1$. Thus,

$$
\bigcup_{m=0}^{q-1} \Phi^{m}\left(\triangle_{j}\right)=\left(\bigcup_{j=0}^{p} \triangle_{j}\right) \bigcup\left(\bigcup_{j=p+1}^{q-1} \triangle_{j}\right)=I_{0} \bigcup I_{1} .
$$

Therefore, there are $p$ iterations on $I_{0}$ and $(q-p)$ iterations on $I_{1}$.

Lemma 1 and remarks 3.4 complete the proof of Theorem 1.

Hence, the lenght $T_{s}$ of this period is established.

\subsubsection{Equivalent bursting orbits}

Definition 3.1. Two periodic bursting orbits are called equivalent if their trajectories have the same period $T_{s}$ and differ from each other by only a translation.

In terms of bursting orbit of a Poincaré map, two orbits are said to be equivalent if they have the same period $q$, with $\alpha=\frac{p}{q}$, and differ from each other up to a phase shift. It means that one could put the initial condition anywhere on the intervall $[0,1[$ and obtain equivalent orbits.

Now, define a matrix $A=\left(a_{m j}\right)_{0 \leq m, j \leq q-1}$, where $a_{m j}$ is the index of the interval $\triangle_{a_{m j}}$, i.e. $\Phi^{m}\left(\triangle_{j}\right)=$ $\triangle_{a_{m j}}$. The elements of the column $j$ are indices of the intervals $\left(\triangle_{j}, 0 \leq j<q\right)$ and the elements of the row are the numbers of the iterations of the orbits. It follows from Lemma 1 that

(1) If $a_{m_{1} j}=a_{m_{2} j}$, then $m_{1}=m_{2}$.

(2) If $a_{m j_{1}}=a_{m j_{2}}$, then $j_{1}=j_{2}$. 
Lemma 2. For $n<q$, one has

$$
\Phi^{n}\left(\triangle_{j}\right)= \begin{cases}\triangle_{j+n(q-p)} & \text { if } \triangle_{j+m(q-p)} \subset I_{0}, m=0, \ldots, n-1, \\ \triangle_{j+(n-l)(q-p)-l p},(l=1, \ldots, n-1) & \text { if } \triangle_{a_{m j}} \subset I_{k}, k=0 \quad \text { or } \quad 1, \\ \triangle_{j-n p} & \text { if } \triangle_{j-m p} \subset I_{1}, m=0, \ldots, n-1 .\end{cases}
$$

where $\triangle_{a_{m j}}$ is of the form $\triangle_{j+m_{1}(q-p)-m_{2} p}$ and $m_{1}=0, \ldots, n-1, m_{2}=0, \ldots, n-1$.

One can easily prove this lemma by induction and show that $\Phi^{n}\left(\triangle_{j}\right)=\triangle_{j+(n-l)(q-p)-l p}$ holds for $l=0, \ldots, n$, and $n<q$.

Next, it will show how the distribution of bursts takes place along a bursting orbit.

The following proposition proves that $\Phi^{m}\left(\triangle_{i}\right)$ and $\Phi^{m}\left(\triangle_{j}\right)$, for $i \neq j$, do not bounce in the same way around $I_{0}$ and $I_{1}$, and gives the repartion of the bursts in an orbit.

Proposition 2. For each $i<q$ and for each $j<q$, with $i \neq j$, there is $n, n<q$, such that $\Phi^{n}\left(\triangle_{i}\right)$ lies in $I_{0}$ and $\Phi^{n}\left(\triangle_{j}\right)$ lies in $I_{1}$, or $\Phi^{n}\left(\triangle_{i}\right)$ lies in $I_{1}$ and $\Phi^{n}\left(\triangle_{j}\right)$ lies in $I_{0}$. Furthermore, there are exactly $q$ bursting orbits $\left(\zeta_{0}, \Phi^{1}\left(\zeta_{0}\right), \ldots, \Phi^{q-1}\left(\zeta_{0}\right)\right), \zeta_{0} \in \triangle_{j}$, which are equivalent to each other.

Proof. In the case where $\triangle_{i}$ and $\triangle_{j}$ do not belong to the same interval $I_{0}$ or $I_{1}$, that is, $i<p$ and $j \geq p$, take $n=0$.

In the case where $\triangle_{i}$ and $\triangle_{j}$ belong to the same interval, say $I_{0}$, suppose $\Phi^{m}\left(\triangle_{i}\right)=\triangle_{a_{m i}}$ and $\Phi^{m}\left(\triangle_{j}\right)=\triangle_{a_{m j}}$ lie in the same interval $I_{0}$. Then, from lemma 2 , one has $\left(a_{m j}-a_{m i}\right)=(j-i)$ for $m<q$.

It follows that $\Phi^{m}\left(\triangle_{i}\right)$ and $\Phi^{m}\left(\triangle_{j}\right)$ can only visit $(p-(j-i))$ times the interval $I_{0}$. This contradicts the fact that $\Phi^{m}\left(\triangle_{i}\right)=\triangle_{a_{m i}}$ visits $p$ times the interval $I_{0}$ for $m<q$.

One can show in the same manner that there is $n$ such that $\Phi^{n}\left(\triangle_{i}\right) \in I_{1}$ and $\Phi^{n}\left(\triangle_{j}\right) \in I_{0}$.

Remark 3.5. In the above, it has been proved that there is $n_{0}$ such that $\Phi^{n_{0}}\left(\triangle_{i}\right) \in I_{0}$ and $\Phi^{n_{0}}\left(\triangle_{j}\right) \in I_{1}$. In this case, there should be an $n_{1}$ such that $\Phi^{n_{1}}\left(\triangle_{i}\right) \in I_{1}$ and $\Phi^{n_{1}}\left(\triangle_{j}\right) \in I_{0}$. If not, then $\Phi^{m}\left(\triangle_{i}\right)$ and $\Phi^{m}\left(\triangle_{j}\right)$ are in the same interval, $I_{0}$ or $I_{1}$, with $m \neq n_{0}$. Suppose they are in $I_{0}$. Then, for $m \neq n_{0}$, each of $\Phi^{m}\left(\triangle_{i}\right)$ and $\Phi^{m}\left(\triangle_{j}\right)$ visits $p$ times the interval $I_{0}$. Since $\Phi^{n_{0}}\left(\triangle_{i}\right)$ is in $I_{0}, \Phi^{m}\left(\triangle_{i}\right)$ visits $(p+1)$ times the interval $I_{0}$ for all $m<q$. This contradicts the fact that $\Phi^{m}\left(\triangle_{i}\right)$ visits $p$ times the interval $I_{0}$ for $m<q$.

The number of indices $n_{i}$ is an even number.

\subsubsection{Repartition of spikes and bursts in a periodic bursting orbit}

Let $r$ be the residual of euclidian division of $(q-p)$ by $p$ if $(q-p)>p$, or the residual of euclidian division of $p$ by $(q-p)$ if $(q-p)<p$. Then, the repartition of the bursts in a bursting orbit is performed in the following way:

\section{Theorem 2.}

- If $(q-p)>p$, i.e. $\left|I_{1}\right|>\left|I_{0}\right|$, then the $(q-p)$ bursts of $([2 s]+1)$ spikes are distributed in $p$ sets of repetitive bursts of $([2 s]+1)$ spikes, separated by bursts of $([2 s]+2)$ spikes. In these $p$ sets, there are $(p-r)$ sets of $n=\left[\frac{q-p}{p}\right]$ repetitive bursts of $([2 s]+1)$ spikes and $r$ sets of $(n+1)$ repetitive bursts of $([2 s]+1)$ spikes.

- If $(q-p)<p$, then the $p$ bursts are distributed in $(q-p)$ sets of repetitive bursts of $([2 s]+2)$ spikes, separated by bursts of $([2 s]+1)$ spikes. In these $(q-p)$ sets, there are $(q-p-r)$ sets of $n=\left[\frac{p}{q-p}\right]$ repetitive bursts of $([2 s]+2)$ spikes and $r$ sets of $(n+1)$ repetitive bursts of $([2 s]+2)$ spikes.

- If $(q-p)=p$, i.e. $q=2 p$ and $\alpha=1 / 2$, then the periodic bursting orbits are of the form: $([2 s]+1)([2 s]+2)$, which can be repeated periodically. 
Proof. For $\alpha=p / q$, a bursting orbit has $p$ bursts of $([2 s]+2)$ spikes and $(q-p)$ bursts of $([2 s]+1)$ spikes. If $(q-p)>p$, then from euclidian division one can write $(q-p)=p\left[\frac{q-p}{p}\right]+r=(p-r) n+r(n+1)$, where $n=\left[\frac{q-p}{p}\right]$. So, the $(q-p)$ bursts of $([2 s]+1)$ spikes are distributed in $(p-r)$ sets of $n$ bursts and $r$ sets of $(n+1)$ bursts.

In addition, for any $i$, since $p<(q-p)$, if $\Phi^{i}\left(\triangle_{j}\right) \in I_{0}$, then $\Phi^{i+1}\left(\triangle_{j}\right)=\triangle_{j+(q-p)} \in I_{1}$. Thereby, each time when the trajectory passes through the $I_{0}$ interval, it leaves this interval at the next iteration, thus generating a single burst of $([2 s]+2)$ spikes. The same reasoning applies if $(q-p)<p$.

In the case of $(q-p)=p$, one has

$$
\Phi\left(\triangle_{j}\right)=\left\{\begin{array}{l}
\triangle_{j+p} \text { when } \triangle_{j} \subset I_{0}(j \leq p-1), \\
\triangle_{j-p} \text { when } \triangle_{j} \subset I_{1}(j \geq p) .
\end{array}\right.
$$

So, if $\triangle_{j} \in I_{0}$ then $\Phi\left(\triangle_{j}\right)=\triangle_{j+p} \in I_{1}$ and $\Phi^{2}\left(\triangle_{j}\right)=\triangle_{j} \in I_{0}$.

In the same way, if $\triangle_{j} \in I_{1}$ then $\Phi\left(\triangle_{j}\right)=\triangle_{j-p} \in I_{0}$ and $\Phi^{2}\left(\triangle_{j}\right)=\triangle_{j} \in I_{1}$. Therefore, the bursting orbits are of the form: $([2 s]+1)([2 s]+2)$, or equivalently, $([2 s]+2)([2 s]+1)$.

Fig. 11 depicts a periodic bursting trajectory computed for $s=1.3$ and $\alpha=\frac{3}{5}$ with periodic pattern of the form (44343). In this case, $(q-p)<p, n=1$ and $r=1$. So, as can be observed in Fig. 11, there are a set of $(n+1)=2$ bursts of 4 spikes and a set of $n=1$ burst of 4 spikes, each of which is separated by a burst of 3 spikes.

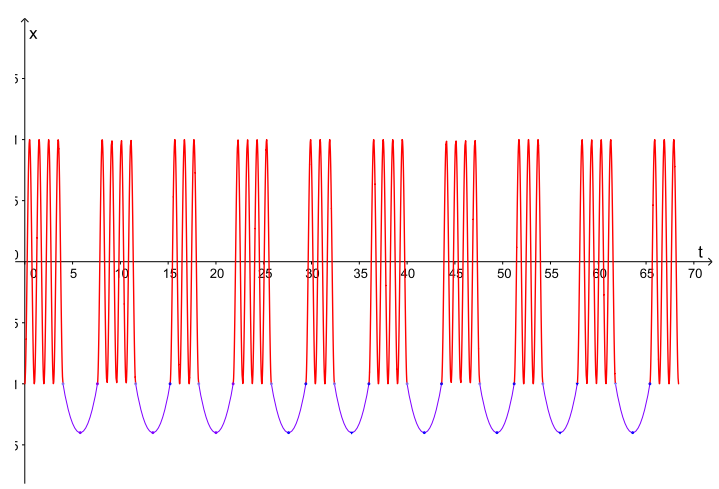

Figure 11. Bursting trajectory with (44343) pattern for $\alpha=\frac{3}{5}$ and $s=1.3$

The next two theorems give the form of the bursting according to whether $p-r>r, p-r<r$ or $p-r=r$, with a specific case of $p-r=1$.

\section{Theorem 3.}

(1) For $\zeta_{0} \in \triangle_{q-1}$, if $p-r>r$, the periodic bursting is distibuted in the following way:

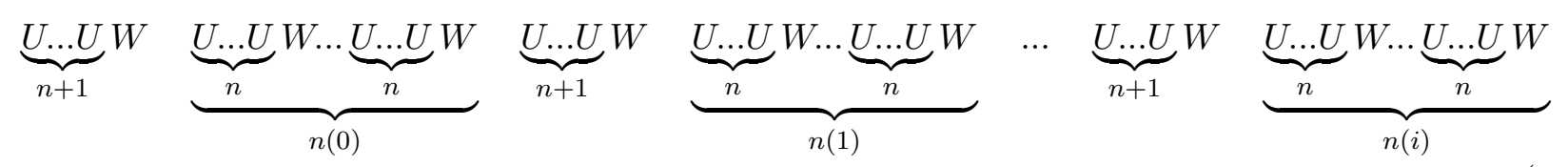

where $U=[2 s]+1, W=[2 s]+2, p-r=n(0) r+r(0), p-(r-r(0))=n(1) r+r(1), p-(r-r(1))=$ $n(2) r+r(2), \ldots, p-(r-r(i-1))=n(i) r+r(i)$, with $n(0)+n(1)+\ldots+n(i)=p-r$ if $r(i)=0$. 
(2) For $\zeta_{0} \in \triangle_{q-1}$, if $p-r<r$, the periodic bursting is distributed in the following way:

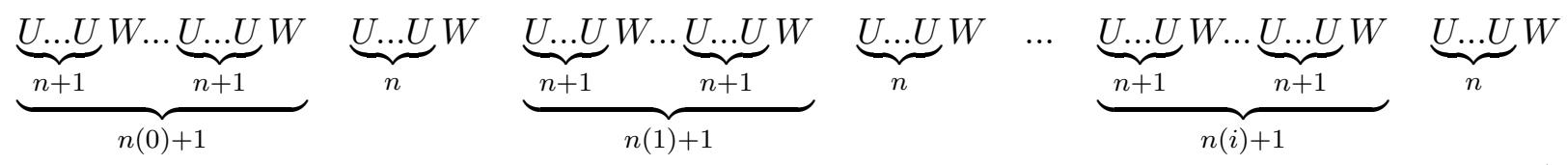

where $r-1=n(0)(p-r)+r(0), r-(p-r)+r(0)=n(1)(p-r)+r(1), \ldots, r-(p-r)+r(i-1)=$ $n(i)(p-r)+r(i)$ and $(n(0)+1)+\ldots+(n(i-1)+1)=r$ if $r(i)=0$ for $i \neq 0$.

Proof. First, perform a bursting for an initial condition $\zeta_{0} \in \triangle_{q-1}$. For the other initial conditions, from Proposition 2, the other periodic bursting are equivalent to this one.

Then, recall that for any $i$, since $p<(q-p)$, if $\Phi^{i}\left(\triangle_{j}\right) \in I_{0}$, then $\Phi^{i+1}\left(\triangle_{j}\right)=\triangle_{j+(q-p)} \in I_{1}$.

(1) If $p-r>r$ i.e. $p>2 r$, then for $\zeta_{0} \in \triangle_{q-1}$ one has

$$
\begin{gathered}
\Phi^{(n+1)}\left(\triangle_{q-1}\right)=\triangle_{r-1}=\left[\frac{r-1}{q}, \frac{r}{q}\left[\subset \left[\frac{r-1}{q}, \frac{p-r}{q}\left[\subset I_{0},\right.\right.\right.\right. \\
\Phi^{(n+1)+1}\left(\triangle_{q-1}\right)=\triangle_{n p+2 r-1}=\left[\frac{n p+2 r-1}{q}, \frac{n p+2 r}{q}\left[\subset \left[\frac{p}{q}, \frac{(n+1) p}{q}\left[\subset I_{1}\right.\right.\right.\right. \\
\text { since } n p+2 r<n p+p .
\end{gathered}
$$

Let $p-r=n(0) r+r(0)$. Then,

$$
\Phi^{[(n+1)+1]+n(0) n+n(0)}\left(\triangle_{q-1}\right)=\triangle_{n p+2 r-1+n(0) r}=\triangle_{(n+1) p+r-r(0)-1}=\triangle_{q-1-r(0)} \quad(r>r(0)) .
$$

Putting $\tilde{n}=[(n+1)+1]+n(0) n+n(0)$, one obtains

$$
\Phi^{\tilde{n}}\left(\triangle_{q-1}\right)=\triangle_{q-1-r(0)} .
$$

Thus, the first part of the bursting is given by

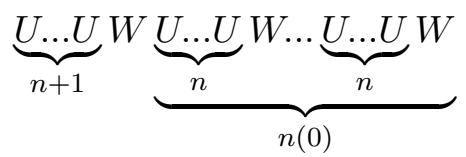

For the next iterations, one has

$$
\Phi^{\tilde{n}+(n+1)}\left(\triangle_{q-1}\right)=\triangle_{r-r(0)-1} \subset I_{0},
$$

and

$$
\Phi^{\tilde{n}+(n+1)+1}\left(\triangle_{q-1}\right)=\triangle_{n p+2 r-r(0)-1}=\triangle_{(n+1) p+r-1-(p-(r-r(0)))}=\triangle_{(n+1) p+r-1-n(1) r-r(1)},
$$

where $p-(r-r(0))=n(1) r+r(1)$. So,

$$
\Phi^{\tilde{n}+(n+1)+1+n(1) n+n(1)}\left(\triangle_{q-1}\right)=\triangle_{(n+1) p+r-r(1)-1} .
$$

Let $\tilde{m}=\tilde{n}+(n+1)+1+n(1) n+n(1)$. Then,

$$
\Phi^{\tilde{m}}\left(\triangle_{q-1}\right)=\triangle_{q-1-r(1)} .
$$

Therefore, the next part of the bursting is

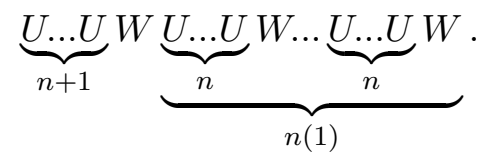

Now, from (40), one can repeat the process as for (28) to obtain the complete periodic bursting. 
(2) If $p-r<r$ i.e. $p<2 r$, then for $\zeta_{0} \in \triangle_{q-1}$, one has

$$
\begin{aligned}
\Phi^{(n+1)}\left(\triangle_{q-1}\right) & =\triangle_{r-1}=\left[\frac{r-1}{q}, \frac{r}{q}\left[\subset \left[\frac{r-1}{q}, \frac{p-r}{q}\left[\subset I_{0},\right.\right.\right.\right. \\
\Phi^{(n+1)+1}\left(\triangle_{q-1}\right)=\triangle_{n p+2 r-1} & \subset\left[\frac{(n+1) p}{q}, \frac{(n+1) p+r}{q}\left[\subset I_{1},\right.\right. \\
& \text { since } n p+2 r-1>n p+p-1 .
\end{aligned}
$$

So,

$$
\Phi^{(n(0)+1)(n+1)+n(0)+1}\left(\triangle_{q-1}\right)=\triangle_{n p+r-1-n(0)(p-r)+r} .
$$

Let $r-1=n(0)(p-r)+r(0)$ with $r(0)<r$. Then,

$$
\Phi^{(n(0)+1)(n+1)+n(0)+1}\left(\triangle_{q-1}\right)=\triangle_{n p+r+r(0)} \subset\left[\frac{p}{q}, \frac{(n+1) p}{q}[\text { since } r(0)<r,\right.
$$

and

$$
\Phi^{\tilde{n}}\left(\triangle_{q-1}\right)=\triangle_{n p+r+r(0)},
$$

for $\tilde{n}=(n(0)+1)(n+1)+n(0)+1$. The first part of the periodic bursting is then given by

$$
\underbrace{\underbrace{U \ldots U}_{n+1} W \ldots \underbrace{U \ldots U}_{n+1} W}_{n(0)+1} .
$$

For the next, one has

$$
\Phi^{\tilde{n}+n+1}\left(\triangle_{q-1}\right)=\triangle_{(n+1) p+r-(p-r)+r(0)}=\triangle_{(n+1) p+n(1)(p-r)+r(1)},
$$

where $n(1)$ and $r(1)$ are defined by $r-(p-r)+r(0)=n(1)(p-r)+r(1)$. Then,

$$
\Phi^{\tilde{n}+n+1+(n(1)+1)(n+1)+n(1)+1}\left(\triangle_{q-1}\right)=\triangle_{(n+1) p-(p-r)+r(1)}=\triangle_{n p+r+r(1)} .
$$

Putting $\tilde{m}=\tilde{n}+n+1+(n(1)+1)(n+1)+n(1)+1$, one obtains

$$
\Phi^{\tilde{m}}\left(\triangle_{q-1}\right)=\triangle_{n p+r+r(1)} .
$$

So, the next part of the bursting is

$$
\underbrace{U \ldots U}_{n} W \underbrace{\underbrace{U \ldots U}_{n+1} W \ldots \underbrace{U \ldots U}_{n+1} W}_{n(1)+1}
$$

Then, from (40), one can repeat the precedent process to give the complete periodic bursting.

As an example, consider the following two cases:

(1) For $p=7, q=17$, there are 7 busts of 4 spikes and $q-p=10$ bursts of 3 spikes, $n=1, r=3$, $p-r=4>r, r(0)=1, n(0)=n(1)=1, r(1)=2, n(2)=2$ and $r(2)=0$. Thus, the periodic bursting is given by (33434334343343434) (see Fig. 12).

(2) For $p=5, q=13$, there are 5 bursts of 4 spikes and $q-p=8$ bursts of 3 spikes, $n=1, r=3$, $p-r=2<r, n(0)=1, r(0)=0, n(1)=0, r(1)=1, n(2)=0$ and $r(2)=0$. The bursting orbit has the pattern (3343343433434) (see Fig. 13).

Remark 3.6. For the case of $p-r=r$, i.e. $p=2 r, q=(n+1) p+r=(2 n+3) r$ and $\operatorname{pgcd}(p, q)=r \neq 1$ for $r \neq 1$. If $r=1$, then the case of $p-r=r$ is included in the general case of $p=r+1$. 


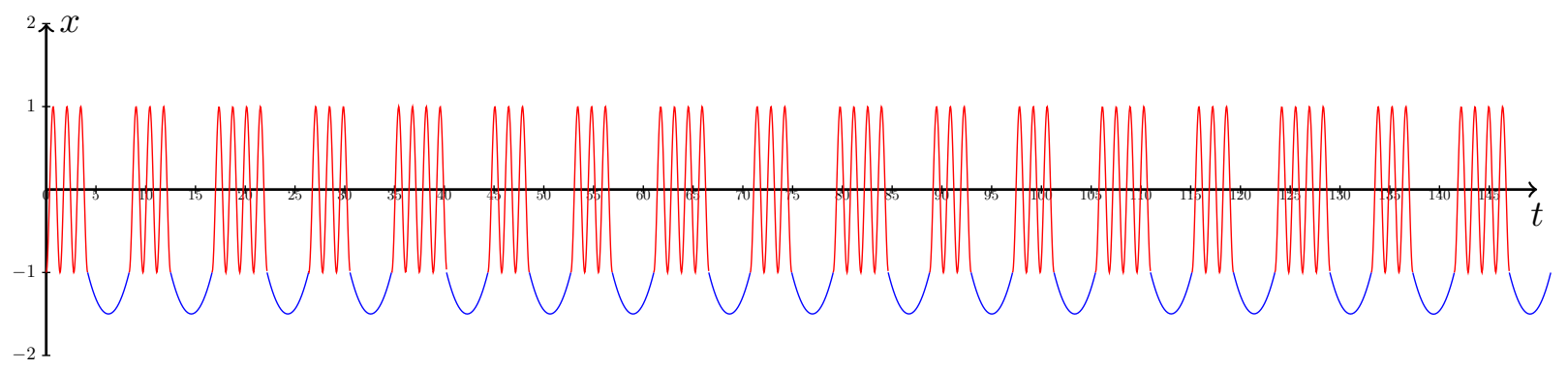

Figure 12. Bursting trajectory with (33434334343343434) pattern for $\alpha=7 / 17$ and $p-r>r$

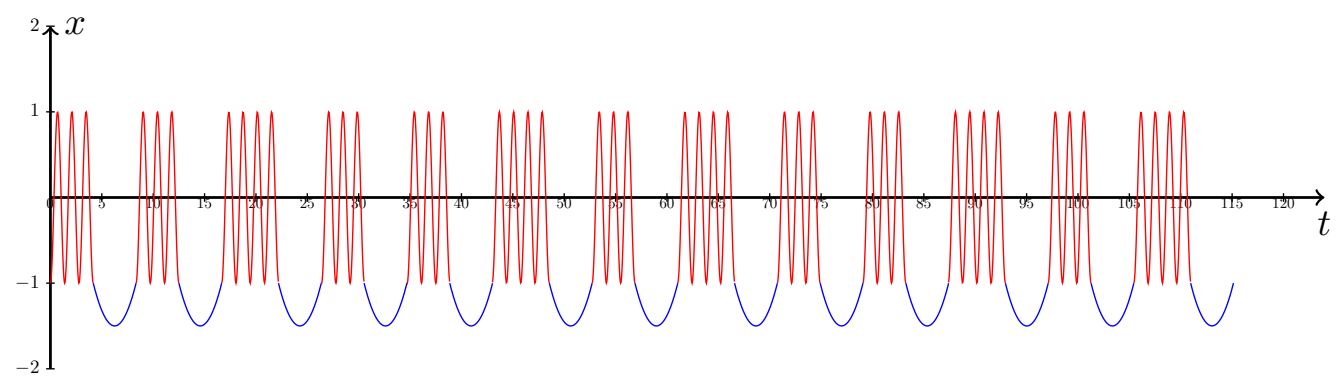

Figure 13. Bursting trajectory with (3343343433434) pattern for $\alpha=5 / 13$ and $p-r<r$

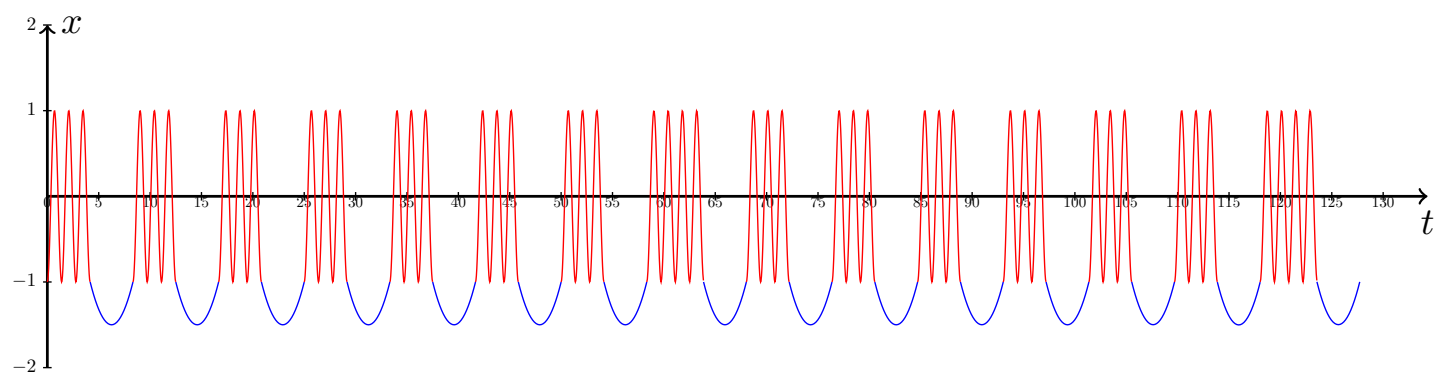

Figure 14. Bursting trajectory with (333333343333334) pattern for $\alpha=2 / 15$ and $p=r+1$

Theorem 4. For $\zeta_{0} \in \triangle_{q-1}$, if $p=r+1$, then the periodic bursting is distributed in the following way:

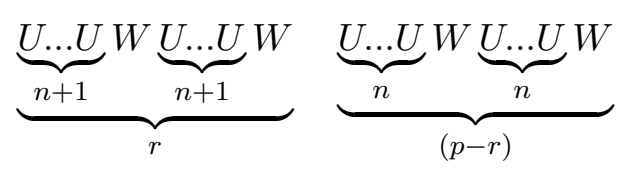

Proof. For $\zeta_{0} \in \triangle_{q-1}$, one has

$$
\Phi^{(n+1)}\left(\triangle_{q-1}\right)=\triangle_{r-1} \subset I_{0},
$$

and

$$
\Phi^{r(n+1)+r}\left(\triangle_{q-1}\right)=\triangle_{(n+1) p+r-1-r(p-r)}=\triangle_{(n+1) p-1}=\triangle_{n p+r} \subset I_{1} .
$$

Thus, the first part of the bursting is 


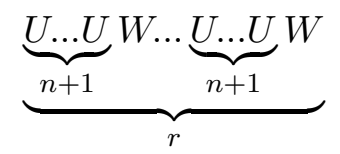

Next, let $\tilde{n}=r(n+1)+r$. Then,

$$
\Phi^{\tilde{n}+n(p-r)+(p-r)}\left(\triangle_{q-1}\right)=\triangle_{(n+1) p+r-1}=\triangle_{q-1} .
$$

So, the $p-r$ remaining bursts are distributed in the following way:

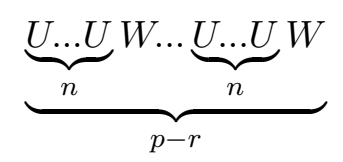

Consider an example for this case. For $p=2, q=15$, there are 2 busts of 4 spikes and $q-p=13$ bursts of 3 spikes, $n=6, r=1, p-r=1$. Thus, the periodic bursting is (3333333433333334) (see Fig. 14).

\subsection{Quasi-periodic bursting orbits}

When $\alpha$ is an irrational number, since the application of $\Phi$ is the translation $\zeta \rightarrow \zeta+\alpha$ (mod 1$)$, each orbit is everywhere dense. Each point in $[0,1[$ has an infinite orbit and at each iteration a new point reaches on the interval $[0,1$ [ so giving rise to a "uniform distribution" on the interval, in the sens that these points are landing, in an ordered manner, in $I_{0}=\left[0, \alpha\left[\right.\right.$ or $I_{1}=[\alpha, 1[$, depending on whether they belong to either of the two intervals. No point is ever revisited in finite time, thus each orbit consists of a quasi periodic bursting.

Let $I$ be an interval $\in\left[0,1\left[\right.\right.$ and $F(x, n)=\#\left\{1 \leq k \leq n / \Phi^{k}(x) \in I\right\}$, where "\#" denotes the number of elements in a set. Then, from Weyl's Theorem [H. Weyl, 1916] below, for $n$ large enough, the average number of points lying in the interval $I$ is equal to the length of this interval. Hence, for each $x$ in $[0,1[$, the iterations $\Phi^{n}(x)$ are uniformly distributed $(\bmod 1)$, that is, the proportion of points lying in the intervals $I_{0}$ and $I_{1}$ is respectively $\alpha$ and $1-\alpha$.

Theorem 5. (Weyl 1916) Let I be an interval of the form $[c, d]$, with $0 \leq c<d \leq 1$. Then, one has $\lim _{n \rightarrow+\infty} \frac{F(x, n)}{n}=c-d$

Fig. 15 and Fig. 16 show respectively the iterates of the map $\Phi$ with the irrational number $\alpha=\frac{1}{\pi}$ and $\alpha=\frac{1}{\sqrt{2}}$. The red circle represents the burst of $([2 \mathrm{~s}]+1)$ spikes and the blue one the burst of $([2 \mathrm{~s}]+2)$ spikes. The number $n$ below or above each circle represents the $n$-th burst.

It can be observed that, when the number of iterations increases, the distance between two bursts decreases and the bursts become closer and closer to each other (see for instance bursts 2 and 24 in Fig. 15 and bursts 7 and 48 in Fig. 16).

When the number of iteration increases, the burst of $([2 \mathrm{~s}]+1)$ spikes will fill up all the interval $I_{1}$ and the one of $([2 \mathrm{~s}]+2)$ spikes will fill up all the interval $I_{0}$.

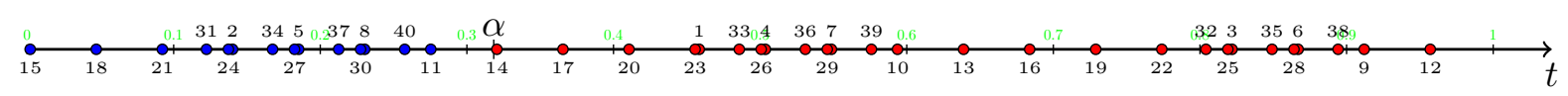

Figure 15. Irrational bursting trajectory for $\alpha=1 / \pi$ 


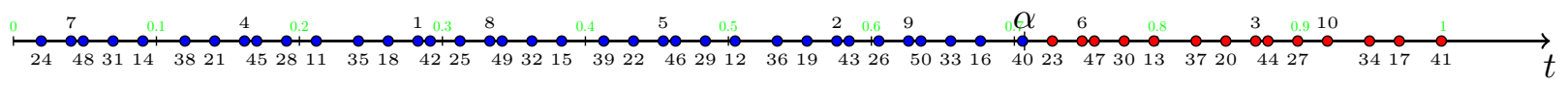

Figure 16. Irrational bursting trajectory for $\alpha=1 / \sqrt{2}$

\section{Chaotic bursting oscillations}

Dynamics on the ribbon is a key to shape the nature of solutions. Comparing Fig. 1 and Fig. 2, it is obvious that, on the ribbon, solutions are spreading in different ways before reaching the cylinder.

Now, consider the dynamics different from those defined in Sec. 2, which allows the onset of chaotic bursting oscillations. These chaotic bursting orbits display mixing of bursts with $U$ and $W$ spikes.

\subsection{Chaotic dynamics on the ribbon}

Keeping the same notation of Sec. 2, on each half-plane $H_{m}$, the parabolic trajectory parametrized by $\theta$ is given by

$$
\left\{\begin{array}{l}
\tilde{X}_{m}(\theta)=\frac{M \theta}{T}(2-\theta) \\
\tilde{Z}_{m}(\theta)=-\frac{(2 s+1)}{2 T} \theta-2 z_{m}^{r}+3 s+1 \text { for } z_{m}^{r} \in\left[s, s+\frac{1}{2}[,\right. \\
\tilde{Z}_{m}(\theta)=-\frac{(2 s+1)}{2 T} \theta+2 z_{m}^{r}-s-1 \text { for } z_{m}^{r} \in\left[s+\frac{1}{2}, s+1[.\right.
\end{array}\right.
$$

\subsection{Poincaré return map}

The half-Poincare map $\Psi_{2}$ on the ribbon is the symmetric tent map. Then, the map $\Phi=\Psi_{2} \circ \Psi_{1}$ is given by

If $\alpha \leq \frac{1}{2}$

$$
\Phi(\zeta)= \begin{cases}-2 \zeta+2 \alpha & \text { if } \zeta \in[0, \alpha[ \\ 2 \zeta-2 \alpha & \text { if } \zeta \in\left[\alpha, \alpha+\frac{1}{2}[\right. \\ -2 \zeta+2 \alpha+2 & \text { if } \zeta \in\left[\alpha+\frac{1}{2}, 1[\right.\end{cases}
$$

(see Fig. 17).

If $\alpha \geq \frac{1}{2}$,

$$
\Phi(\zeta)= \begin{cases}2 \zeta+2(1-\alpha) & \text { if } \zeta \in\left[0, \alpha-\frac{1}{2}[,\right. \\ -2 \zeta+2 \alpha & \text { if } \zeta \in\left[\alpha-\frac{1}{2}, \alpha[\right. \\ 2 \zeta-2 \alpha & \text { if } \zeta \in[\alpha, 1[.\end{cases}
$$

(see Fig. 20).

This proves that $\Phi$ has a horseshoe on the interval $I$.

Let $J$ be a closed interval of $I$. Suppose that there are two disjoint closed intervals, $K_{0} \subset J \cap I_{0}$ and $K_{1} \subset J \cap I_{1}$, and the restriction of $\Phi$ to each $K_{i}$, i.e., $\Phi \mid K_{i}$, is monotonic and continuous, such that $\Phi\left(K_{j}\right) \supset \cup_{i=0}^{1} K_{i}, j=0,1$.

Let

$$
\begin{aligned}
& \Sigma=\left\{\left(t_{0}, t_{1}, t_{2}, \cdots\right), t_{j}=0 \quad \text { or } \quad t_{j}=1\right\} \\
& \Lambda=\left\{\zeta \in I: \Phi^{n}(\zeta) \in I \quad \forall n \geq 0\right\}
\end{aligned}
$$

and the shift map $\sigma: \Sigma \rightarrow \Sigma$ defined by $\sigma\left(t_{0} t_{1} t_{2} \cdots\right)=\left(t_{1} t_{2} t_{3} \cdots\right)$. 
Recall ([Devaney, 2003]) that there exists a compact invariant set $\Lambda \subset J$, such that $\Phi \mid \Lambda$ is semiconjugate to the shift map $\sigma$ on $\Sigma$ via a continuous and onto map $h: \Sigma \longrightarrow \Lambda$, where

$$
\Lambda=\cap_{i=0}^{\infty} \Phi^{-i}\left(K_{1} \cup K_{0}\right)=\cup_{\left(t_{0} t_{1} t_{3} \cdots\right) \in \Sigma} \cap_{i=0}^{\infty} \Phi^{-i}\left(K_{t_{i}}\right) .
$$

One has

$$
\Phi^{n} \circ h=h \circ \sigma^{n} .
$$

So, $h$ converts $\sigma$-orbits to $\Phi$-orbits. Therefore, $\Phi$-orbits are in one-to-one correspondence with $\sigma$-orbits. In particular, if $t$ is a periodic point for $\sigma$, then $h(t)$ is a periodic point for $\Phi$ with the same period. If there is an eventually periodic point for $\sigma$, then $h$ gives an analogous point for $\Phi$. Indeed, $h$ converts the dynamical behavior observed for $\sigma$. Thus, the dynamics of $\sigma$ on $\Sigma$ and $\Phi$ on $\Lambda$ are essentially the same.

For any $\zeta_{0} \in \Lambda$, there is $t_{0}=\left(t_{0}(0) t_{0}(1) t_{0}(2) \cdots\right) \in \Sigma$ such that $\Phi^{n-1}\left(\zeta_{0}\right) \in K_{t_{0}(n-1)} \subset I_{t_{0}(n-1)}$. The point $\left(\Phi^{n}\left(\zeta_{0}\right)-(2 s+1)\right)$ denotes the point where the bursting orbit begins to turn around the cylinder to form the burst $B_{n}$. The number of spikes within the burst $B_{n}$ is

$$
\left.t_{0}(n-1)(2[s]+1)+\left(1-t_{0}(n-1)\right)([2 s]+2)\right),
$$

that is, the number of spikes within the burst $B_{n}$ is $([2 s]+2)$ if $\Phi^{n-1}\left(\zeta_{0}\right)$ lies in $K_{0}\left(t_{0}(n-1)=0\right)$ and $([2 s]+1)$ if $\Phi^{n-1}\left(\zeta_{0}\right)$ lies in $K_{1}\left(t_{0}(n-1)=1\right)$.

The map $\sigma$ has countably infinitely many of periodic orbits consisting of the orbits of all periods, and for any $n>0, \sigma$ has $\left(2^{n}-2\right)$ periodic points of period $n$.

Let $t_{i}=\left(t_{i}(0) t_{i}(1) \cdots t_{i}(n-1) t_{i}(0) t_{i}(1) \cdots t_{i}(n-1) \cdots\right)$ be a periodic point of period $n$ for $\sigma$. Then, there is $\zeta_{0} \in \Lambda$ such that $h\left(t_{i}\right)=\zeta_{0}$ is a periodic point of period $n$ for $\Phi$ with $\Phi^{m}\left(\zeta_{0}\right) \in K_{t_{i}(m)}$ for any $m<n$ and $\Phi^{n}\left(\zeta_{0}\right)=\zeta_{0} \in K_{t_{i}(0)}$. There is a bursting orbit of period

$$
T_{i}=a_{i}([2 s]+2)+b_{i}([2 s]+1)+\sum_{j=0}^{n-1} \beta_{i, j},
$$

with $a_{i}+b_{i}=n$, where $a_{i}=\sharp\left\{j \leq n-1, t_{i}(j)=0\right\}$ is the number of bursts of $([2 s]+2)$ spikes and $b_{i}=\sharp\left\{j \leq n-1, t_{i}(j)=1\right\}$ is the number of bursts of $([2 s]+1)$ spikes, and $\beta_{i, j}$ denotes the time it takes for the solution to form the period of quiescence $j<n-1$ and then return to the cylinder.

Therefore, for each period $n$ of $\sigma$, there are $\left(2^{n}-2\right)$ types of periodic bursting of periods $T_{i}, 1 \leq i \leq$ $\left(2^{n}-2\right)$, with all possible distributions of numbers of spikes $([2 s]+2)$ and $([2 s]+1)$ per bursts.

Proposition 3. For $\alpha<\frac{1}{2}$, $\Phi$ has horseshoes on the intervals $[0,2 \alpha]$ and $[2 \alpha, 1]$. In the interval $[0,2 \alpha]$, there are $K_{0}=[0, \alpha] \in I_{0}$ and $[\alpha, 2 \alpha] \in I_{1}$ and $\Phi([0,2 \alpha])=[0,2 \alpha]$, and in the interval $[2 \alpha, 1], \Phi([2 \alpha, 1])=[2 \alpha, 1]$.

In the case of $\alpha>\frac{1}{2}$, for $\alpha=0.75, \Phi^{2}$ has a horseshoe on the interval $[0,0.5]$ with $\Phi^{2}([0,0.5]) \subset$ $[0,0.5] \subset I_{0}$ and a horseshoe on the interval $[0.5,1]$ with $\Phi^{2}([0.5,1]) \subset[0.5,1]$.

Remark 4.1. For $\alpha<\frac{1}{2}$, for initial conditions in the interval $[0,2 \alpha]$, there are burstings with a mix of $(2[s]+1)$ and $([2 s]+2)$ bursts (see Fig. 18$)$. For initial conditions in the interval $[2 \alpha, 1] \subset I_{1}$, there is bursting with only $(2[s]+1)$ bursts (see Fig. 19).

For $\alpha=0.75, \Phi^{2}([0,0.5]) \subset I_{0}$, so there is bursting with only $([2 s]+2)$ bursts (see Fig. 21$)$ and in the interval $[0.5,1]$, there is bursting with a mix of $(2[\mathrm{~s}]+1)$ and $([2 \mathrm{~s}]+2)$ bursts (see Fig. 22).

Proof. For $\alpha<\frac{1}{2}$ (see Fig. 17 for $\alpha=1.8$ ), one has $\Phi(2 \alpha)=2 \alpha$, so $\Phi([0,2 \alpha])=[0,2 \alpha]$ and $\Phi([2 \alpha, 1])=$ $[2 \alpha, 1]$. For $\alpha=0.75,2-2 \alpha=0.5$, one has $\Phi^{2}(0)=0.5$ and $\Phi^{2}(0.5)=0.5$. 


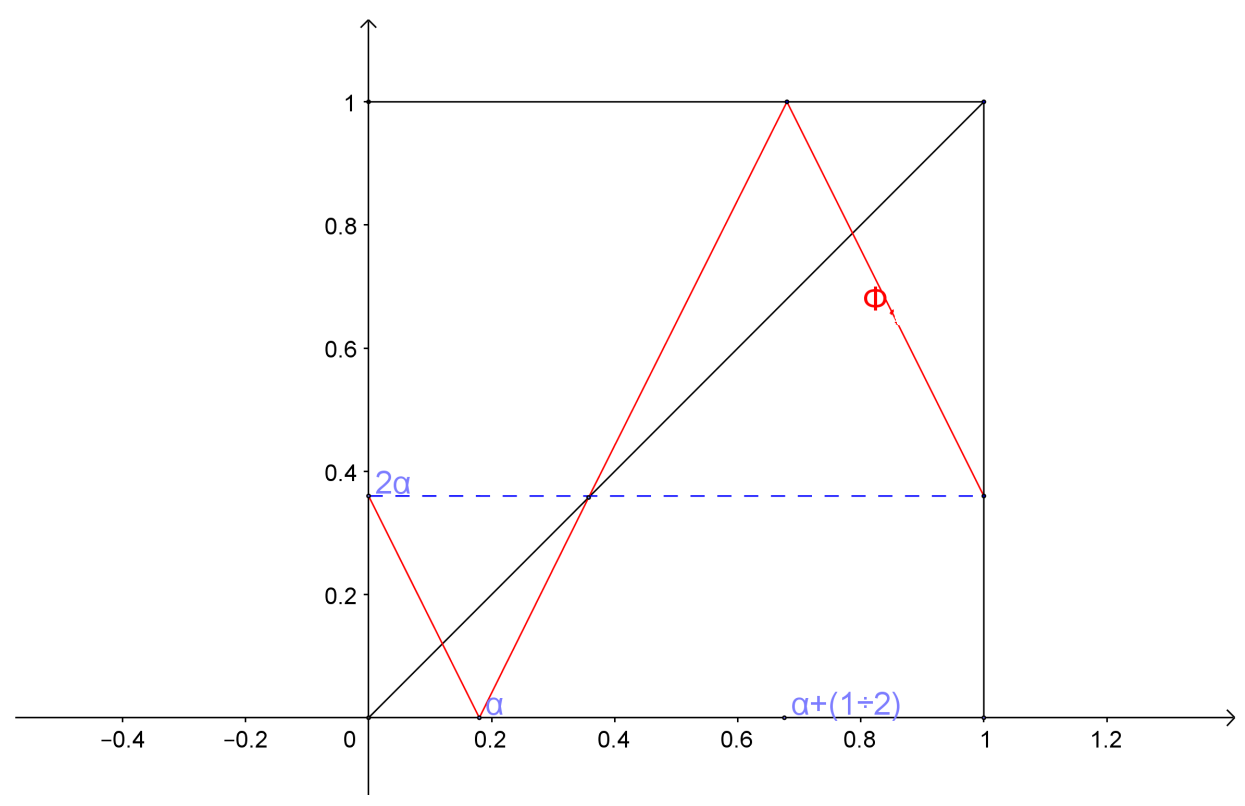

Figure 17. Graph of the Poincaré map $\Phi$ for $\alpha<\frac{1}{2}$.

\section{Geometrical mug-shaped model: The general case}

In previous sections, the simplest case of the geometrical mug-shaped model has been thoroughly studied. In this simplest case, bursting oscillations are mixing of bursts of $U$ and $W$ spikes. However, it is possible to model bursting oscillations which mix bursts of any number of spikes. In this section, an example of the extension of the simplest case model is given to demonstrate this possibility. In this example, oscillations mix bursts of 4 to 8 spikes.

The main idea to extend the simplest model is to split the interval of injection into several intervals, while leaving the interval of reinjection unchanged.

\subsection{Split interval of injection}

To explain the extended geometrical model using an example (Fig. 23), let $s=1.4$ and the interval of injection be a union of four subintervals:

$I_{s}^{r}=[2.1,2.4[\cup[2.4,2.5[\cup[3.8,4.1[\cup[5.5,5.8[$.

When a trajectory on the cylinder reaches for $x=-1$ the subinterval $[1.4,2.1$ [, it cannot escape to the ribbon and must continue to turn around the cylinder, except when it reaches the first subinterval [2.1, 2.4[. The trajectory which continues on the cylinder can eventually escape through the window of the second subinterval $[2.4,2.5[$ or the next one $[3.8,4.1[$ or $[5.5,5.8[$.

The interval of reinjection has the same definition as for the simplest model case:

$I_{s}^{c}=[-2.4,-1.4[$.

Keeping the same notation of Sec. 2, on each half-plane $H_{m}$, the parabolic trajectory is always parametrized by $\theta$ following Eq. (14).

$$
\left\{\begin{array}{l}
\tilde{X}_{m}(\theta)=\frac{M \theta}{T}(2-\theta), \\
\tilde{Z}_{m}(\theta)=-\frac{3.8}{2 T} \theta+z_{m}^{r} \quad \text { for } \quad z_{m}^{r} \in[2.1,2.4[, \\
\tilde{Z}_{m}(\theta)=-\frac{4.8}{2 T} \theta+z_{m}^{r} \quad \text { for } \quad z_{m}^{r} \in[2.4,2.5[, \\
\tilde{Z}_{m}(\theta)=-\frac{5.8}{2 T} \theta+z_{m}^{r} \quad \text { for } \quad z_{m}^{r} \in[3.8,4.1[, \\
\tilde{Z}_{m}(\theta)=-\frac{7.8}{2 T} \theta+z_{m}^{r} \quad \text { for } \quad z_{m}^{r} \in[5.5,5.8[.
\end{array}\right.
$$




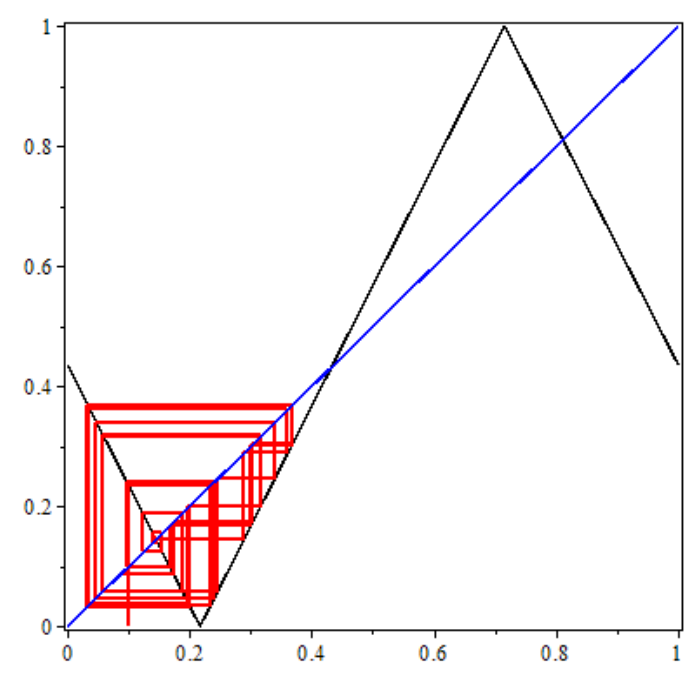

Figure 18. Graphical iterations for the map $\Phi$ when $\alpha<\frac{1}{2}$ and for initial condition $\zeta_{0}=0.1$

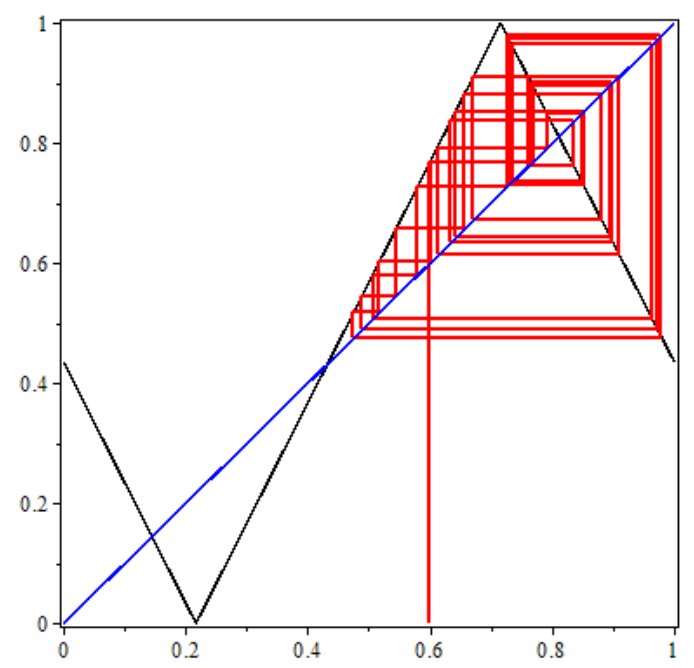

Figure 19. Graphical iterations for the map $\Phi$ when $\alpha<\frac{1}{2}$ and for initial condition $\zeta_{0}=0.6$

Therefore, the half-Poincaré map $\Psi_{2}$ is defined as

$$
\left\{\begin{array}{lll}
\Psi_{2}(z)=z-(2 s+1) & \text { for } & z_{m}^{r} \in[2.1,2.4[, \\
\Psi_{2}(z)=z-(2 s+2) & \text { for } & z_{m}^{r} \in[2.4,2.5[, \\
\Psi_{2}(z)=z-(2 s+3) & \text { for } & z_{m}^{r} \in[3.8,4.1[, \\
\Psi_{2}(z)=z-(2 s+5) & \text { for } & z_{m}^{r} \in[5.5,5.8[.
\end{array}\right.
$$

Figure 23 displays a first periodic orbit with initial point $z=-2.1$.

This orbit intersects the border of the cylinder $x=\{-1\}$ for the following values of $z:-2.1 \rightarrow-1.1 \rightarrow$ $-0.1 \rightarrow 0.9 \rightarrow 1.9 \rightarrow 2.9 \rightarrow 3.9$ and escapes from the cylinder to the ribbon from the subinterval [3.8, 4.1[ after 6 spikes.

After that, the orbit is back on the cylinder at the point $z=-1.9$ and intersects again the border of the cylinder $x=\{-1\}$ for the following values of $z:-1.9 \rightarrow-0.9 \rightarrow 0.1 \rightarrow 1.1 \rightarrow 2.1$ escaping to the ribbon after 4 spikes from the subinterval [2.1,2.4[, and so on, being again on the cylinder for $z=-1.7 \rightarrow-0.7 \rightarrow 0.3 \rightarrow 1.3 \rightarrow 2.3$ escaping again from the subinterval [2.1,2.4[, after 4 spikes to the ribbon and then on the cylinder for $z=-1.5 \rightarrow-0.5 \rightarrow 0.5 \rightarrow 1.5 \rightarrow 2.5 \rightarrow 3.5 \rightarrow 4.5 \rightarrow 5.5$ escaping 


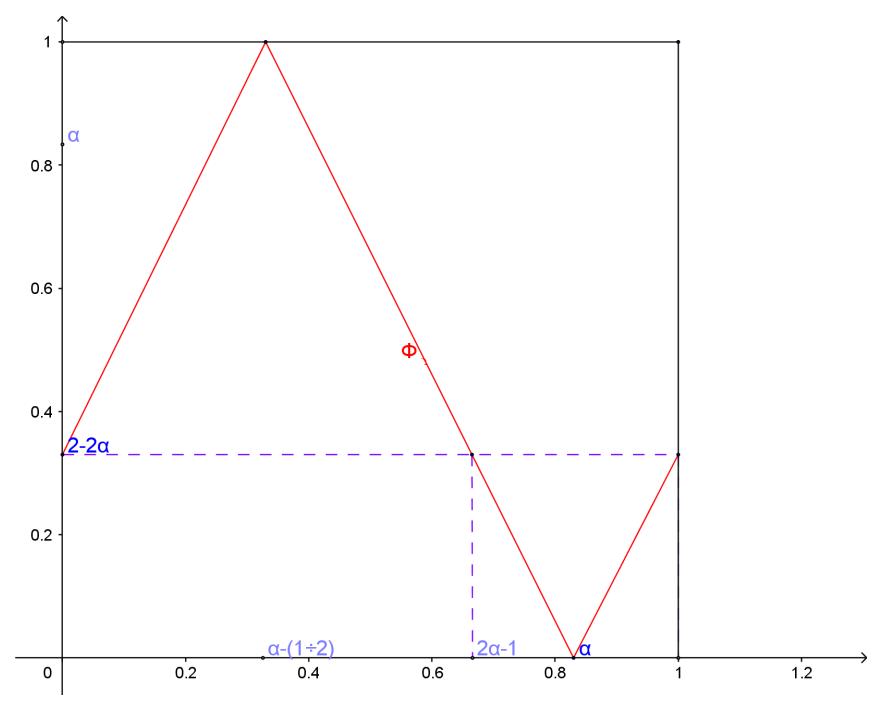

Figure 20. Graph of the Poincaré map $\Phi$ for $\alpha>\frac{1}{2}$.

again from the subinterval $[5.5,5.8[$ after 7 spikes, being one more time on the cylinder for $z=-2.3 \rightarrow$ $-1.3 \rightarrow-0.3 \rightarrow 0.7 \rightarrow 1.7 \rightarrow 2.7 \rightarrow 3.7 \rightarrow 4.7 \rightarrow 5.7$ escaping one more time from the subinterval $[5.5,5.8[$ after 8 spikes and returning to the initial value $z=-2.1$. For this periodic orbit, the bursts are successively containing $6,4,4,7$ and 8 spikes.

\subsection{Coexisting periodic solutions}

More interestingly in this example, two periodic solutions with different patterns can coexist. The second periodic orbit starts with the initial point $z=-1.6$, and has the following pattern:

$-1.6 \rightarrow-0.6 \rightarrow 0.4 \rightarrow 1.4 \rightarrow 2.4$ exit $[2.4,2.5[$ after 4 spikes,

$-2.4 \rightarrow-1.4 \rightarrow-0.4 \rightarrow 0.6 \rightarrow 1.6 \rightarrow 2.6 \rightarrow 3.6 \rightarrow 4.6 \rightarrow 5.6$ exit $[5.5,5.8[$ after 8 spikes,

$-2.2 \rightarrow-1.2 \rightarrow-0.2 \rightarrow 0.8 \rightarrow 1.8 \rightarrow 2.8 \rightarrow 3.8$ exit $[3.8,4.1[$ after 6 spikes,

$-2 \rightarrow-1 \rightarrow 0 \rightarrow 1 \rightarrow 2 \rightarrow 3 \rightarrow 4$ exit $[3.8,4.1[$ after 6 spikes,

$-1.8 \rightarrow-0.8 \rightarrow 0.2 \rightarrow 1.2 \rightarrow 2.2$ exit $[2.1,2.4$ [ after 4 spikes and back to the initial value $z=-1.6$.

The bursts are composed of 4,8,6,6, 4 spikes.

The above two examples of coexisting periodic bursting orbits, exhibiting different patterns, show the extraordinary versatility of this generalized model.

Depending on the shape of the injection interval, more coexisting bursting orbits, either periodic of quasiperiodic, could coexist.

\section{Conclusion}

In this paper, a simplest bursting neuron model is introduced, based on the Hodgkin-Huxley and HindmarshRose models, with a geometric mug-shaped branched manifold, which can mix burst of $n$ and $n+1$ spikes. The model can generate complex dynamic patterns, including periodic and quasiperiodic orbits. Rigorous analytic proofs of their existence are given, and their complex dynamics are discussed with clear graphical demonstrations. Furthermore, a more complex case of the model, which can generate chaotic bursting orbits with $n$ and $n+1$ spikes, is established and discussed. The model is generalized to obtain mixing burst of any number of spikes. Finally, an example with bursts of 4 to 8 spikes is demonstrated. The advantage of the new model is its simplicity in form with complex dynamics, presenting most neuron burst patterns for complete analysis. 


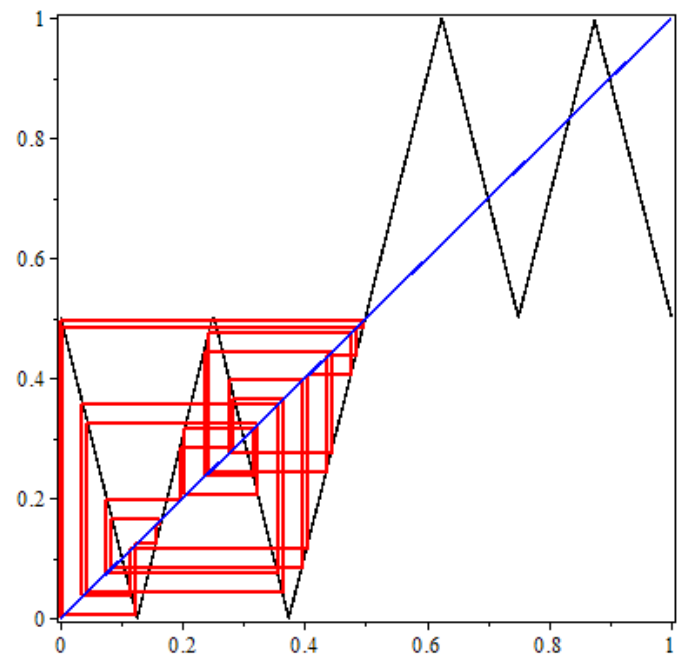

Figure 21. Graphical iterations for the map $\Phi^{2}$ when $\alpha=0.75$ and for initial condition $\zeta_{0}=0.001$

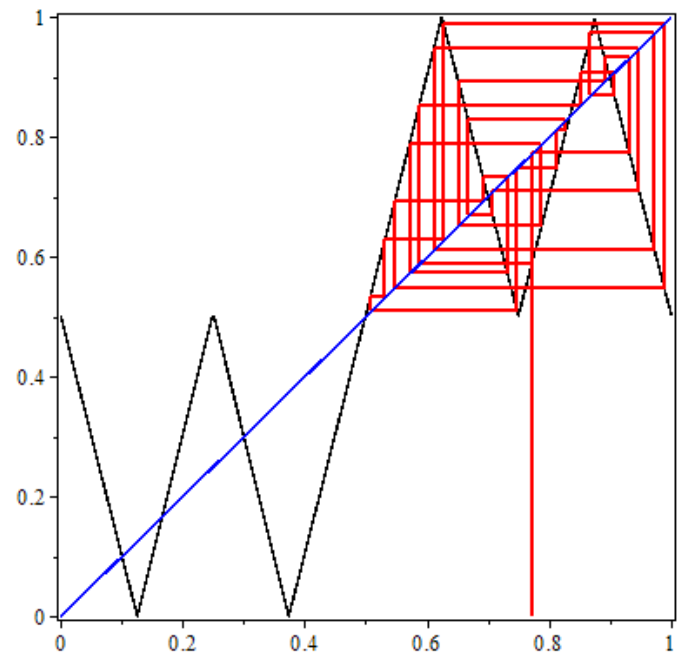

Figure 22. Graphical iterations for the map $\Phi^{2}$ when $\alpha=0.75$ and for initial condition $\zeta_{0}=0.772$

\section{References}

Abdelouahab, M.-S., Lozi, R., Chen, G. [2019] "Complex canard explosion in a fractional-order FitzHughNagumo model", International Journal of Bifurcation and Chaos, 29, No. 8, 1950111 (22 pages).

Corson, N. [2009] Dynamique d'un modèle neuronal, synchronisation et complexité, thèse de Doctorat d'Etat, (Université du Havre).

Devaney R. L. [2003] An Introduction to Chaotic Dynamical Systems, Addison-Wesley Publishing Company, Inc.

Hindmarsh, J. L., Rose, R. M. [1982] "A model of the nerve impulse using two first-order differential equations," Nature. 296, pp. 162-164.

Hindmarsh, J .L., Rose, R. M. [1984] "A model of neuronal bursting using three coupled first order differential equations," Proc. R. Soc. London B. 221, pp. 87-102.

Hodgkin, A. L. \& Huxley, A. F. [1952] "A quantitative description of membrane current and its application to conduction and excitation in nerve," J. Physiol. 117, pp. 500-544.

Shilnikov, A. L. \& Rulkov, N. F. [2003] "Origin of chaos in a two-dimensional map modelling spiking-bursting neural activity," International Journal of Bifurcation and Chaos. 13, pp. 3325-3340. 


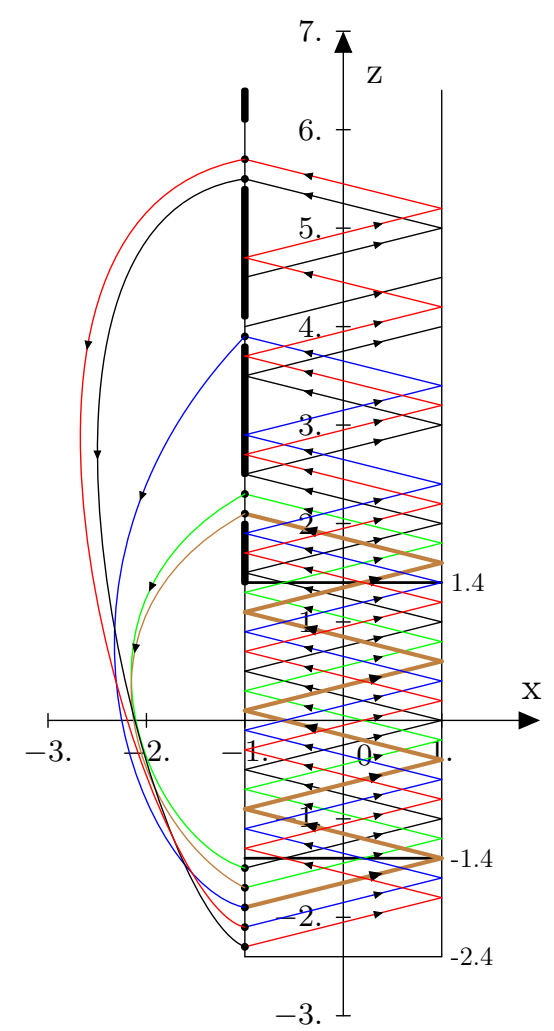

Figure 23. Periodic orbit of the generalized mug-shaped model for $s=1.4$, initial value $z=-2.1$.

Shilnikov, A. L. \& Kolomiets, M. [2008] "Methods of the qualitative theory for the Hindmarsh-Model: A case study.A Tutorial,"International Journal of Bifurcation and Chaos. 18, pp. 2141-2168.

Izhikevich, E. M. [2007] Dynamical Systems in Neuroscience: The Geometry of Excitability and Bursting, The MIT Press Cambridge, Massachusetts, London, England.

Stiles, J. \& Jernigan, T. J. [2010] "The Basics of Brain Development," Neuropsychol. Rev. 4, pp. 327-348.

Weyl, H. [1916] "Uber die Gleichverteilung von Zahlen mod. Eins, Mathematische Annalen, 77, no. 3, pp. 313-352. 